\title{
VECTOR-VALUED POSITIVE DEFINITE FUNCTIONS, THE BERG-MASERICK THEOREM, AND APPLICATIONS
}

\author{
P. RESSEL and W. J. RICKER*
}

\section{Introduction}

The theory of positive definite functions is an important and extensive area of modern mathematics, having many and varied applications. One of the fundamental results is the integral representation theorem for exponentially bounded, positive definite functions due to C. Berg and P. H. Maserick; see [4, Ch. 4, Section 2] and [5]. In order to formulate this result we require some terminology.

Let $S$ be a commutative semigroup with identity element (always denoted by $e$ ) and equipped with an involution $s \mapsto s^{-}$(i.e. $\left(s^{-}\right)^{-}=s$ and $(s t)^{-}=s^{-} t^{-}$ for all $s, t \in S$ ). A character of $S$ is any function $\rho: S \rightarrow$ C satisfying $\rho(e)=1$ and $\rho\left(s t^{-}\right)=\rho(s) \overline{\rho(t)}$ for all $s, t \in S$. The set of all characters of $S$ is denoted by $S^{*}$; it is a completely regular topological space when equipped with the topology of pointwise convergence inherited from $\mathrm{C}^{S}$. Given $s \in S$, the continuous function $\hat{s}: S^{*} \rightarrow \mathrm{C}$ is defined by $\hat{s}(\rho):=\rho(s)$ for $\rho \in S^{*}$.

A scalar function $f: S \rightarrow \mathrm{C}$ is called positive definite if $\sum_{j, k=1}^{n} c_{j} \overline{c_{k}} f\left(s_{j} s_{k}^{-}\right)$ $\geq 0$ for all choices of $n \in \mathrm{N}$, and sets $\left\{s_{j}\right\}_{j=1}^{n} \subseteq S$ and $\left\{c_{j}\right\}_{j=1}^{n} \subseteq \mathrm{C}$. In particular, every character $\rho \in S^{*}$ is positive definite. A function $\alpha: S \rightarrow$ $[0, \infty)$ satisfying $\alpha(e)=1$ is called an absolute value if $\alpha\left(s^{-}\right)=\alpha(s)$ and $\alpha(s t) \leq \alpha(s) \alpha(t)$ for all $s, t \in S$. Then a scalar function $f: S \rightarrow \mathrm{C}$ is called $\alpha$-bounded if there exists a constant $C \geq 0$ such that $|f(s)| \leq C \alpha(s)$ for all $s \in S$. If, in addition, $f$ happens to be positive definite, then it is possible to choose $C=f(e)$. We say that $f$ is exponentially bounded if it is $\alpha$-bounded for some absolute value $\alpha$ on $S$. A character $\rho \in S^{*}$ is $\alpha$-bounded if and only if $|\rho| \leq \alpha$. Hence, the set $S^{\alpha}$ of all $\alpha$-bounded characters is a compact subset of $S^{*}$. For all of these notions and further properties we refer to [4, Ch. 4].

\footnotetext{
* The support of both the Australian Department of Science, Industry and Tourism and the Maximilian Bickhoff Stiftung are gratefully acknowledged.

Received April 13, 1999.
} 
Finally, the set of all non-negative Radon measures defined on the Borel sets $\mathscr{B}\left(S^{*}\right)$ of $S^{*}$ is denoted by $M_{+}\left(S^{*}\right)$. Given any absolute value $\alpha$ on $S$ the subset of $M_{+}\left(S^{*}\right)$ consisting of all elements $\mu$ whose support $\operatorname{supp}(\mu)$ is contained in the compact subset $S^{\alpha} \subseteq S^{*}$ is denoted by $M_{+}\left(S^{\alpha}\right)$. The generalized Laplace transform $\hat{\mu}: S \rightarrow \mathrm{C}$ of such a measure $\mu$ is then defined by $s \mapsto \int_{S^{*}} \hat{s} d \mu$ for each $s \in S$.

Theorem 1.1 (Berg-Maserick Theorem). Let $S$ be a unital, commutative semigroup with an involution and $\alpha: S \rightarrow[0, \infty)$ be an absolute value. Given a positive definite and $\alpha$-bounded function $f: S \rightarrow \mathrm{C}$ there exists a unique measure $\mu \in M_{+}\left(S^{\alpha}\right)$ such that $f=\hat{\mu}$, that is

$$
f(s)=\int_{S^{*}} \hat{s} d \mu, \quad s \in S .
$$

It is a well established "principle" that most integral representation formulae for scalar-valued functions have a vector-valued analogue if suitably formulated; see [33] and the references therein, for example. The aim of this paper is to provide a vector analogue of the Berg-Maserick theorem. What then should be the essential ingredients of such a generalization?

There is already an extensive literature on many aspects of vector-valued positive definite functions; the crucial point is that the quadratic form naturally associated with the function should take its values in a positive cone. In this regard we follow the approach adopted in the Banach space setting by P. L. Falb and U. Haussmann, [12]. Since we have in mind applying our results to the integral representation of certain semigroups of linear operators acting in a Banach space, but with respect to the strong operator topology (see Section 3), our setting is more general than that of [12] and allows for functions with values in a locally convex Hausdorff space (briefly, lcHs). The corresponding vector measure will also be required to assume its values in the same positive cone in which the quadratic form associated with the given function takes its values. It is the notion of $\alpha$-boundedness in the vector setting which is somewhat more subtle to identify. Eventually it should "somehow" lead to the existence of an appropriate vector measure whose generalized Laplace transform is the given function. According to the theory of vector measures, this suggests that weak compactness must enter in some format or other; this is indeed the case and is suitably formulated via Definition 2.4.

So, with the notions of positive definiteness, positive vector measure and $\alpha$-boundedness suitably extended to the setting of a lcHs we establish, in Section 2, a vector-valued Berg-Maserick theorem; see Propositions 2.6 and 2.7. Since the $\alpha$-boundedness of a vector-valued function is the most difficult property to verify in practice, we also present a few relevant criteria which can 
sometimes be used in this regard; see Propositions 2.10, 2.12 and 2.13. An application to additively correlated stochastic processes whose time parameter varies in a semigroup with involution is given at the end of Section 1 . The final section, as indicated above, is devoted to establishing an integral representation formula for positive, exponentially bounded semigroups of scalar operators (in the sense of N. Dunford) acting in Banach spaces; see Propositions 3.1 and 3.7. Indeed, it is this result (generalizing the integral representation of *-representations of normal operators in Hilbert space, [30]) which is one of the main motivations for establishing a vector-valued Berg-Maserick theorem.

\section{A vector-valued Berg-Maserick theorem}

Let $X$ be a (complex) lcHs and $X^{\prime}$ be the continuous dual space of $X$. Given a subset $\Phi \subseteq X^{\prime}$ we let

$$
C_{\Phi}:=\{x \in X:\langle x, \varphi\rangle \geq 0 \text { for all } \varphi \in \Phi\}
$$

be the positive cone determined by $\Phi$. In order to avoid trivialities we always assume that $\Phi$ contains at least one non-zero element. Let $S$ be a unital, commutative semigroup with an involution.

Definition 2.1. A function $f: S \rightarrow X$ is called $\Phi$-positive definite if $\sum_{j, k=1}^{n} c_{j} \bar{c}_{k} f\left(s_{j} s_{k}^{-}\right) \in C_{\Phi}$ for all choices of finitely many elements $\left\{c_{1}, \ldots, c_{n}\right\} \subseteq \mathrm{C}$ and $\left\{s_{1}, \ldots, s_{n}\right\} \subseteq S$.

This definition is equivalent with the requirement that each $\mathrm{C}$-valued scalar function $\langle f, \varphi\rangle: s \mapsto\langle f(s), \varphi\rangle$, for $s \in S$, is positive definite in the usual sense of scalar functions, for every $\varphi \in \Phi$. In the case when $X$ is a Banach space and $S$ is an abelian group with involution the inverse function $s \mapsto s^{-1}$ on $S$, Definition 2.1 agrees with that in [12, p. 605].

Definition 2.1 also accommodates the best known classical example in the vector-valued setting. According to B. Sz.-Nagy [38, Section 6], if $H$ is a Hilbert space, then a function $f$ on $S$ with values in the Banach space $B(H)$ of all continuous linear operators on $H$ is positive definite if $\sum_{j, k=1}^{n}\left\langle f\left(s_{j} s_{k}^{-}\right) h_{j}, h_{k}\right\rangle \geq$ 0 for all choices of finite subsets $\left\{h_{j}\right\}_{j=1}^{n} \subseteq H$ and $\left\{s_{j}\right\}_{j=1}^{n} \subseteq S$. Such a function $f$ is $\Phi$-positive definite in the sense of Definition 2.1 when $\Phi$ is taken to be the set of all continuous linear functionals of the form $T \mapsto\langle T u, u\rangle, T \in B(H)$, for each $u \in H$. On the other hand, if $f$ happens to take its values in an abelian $C^{*}$-algebra and is $\Phi$-positive definite in the sense of Definition 2.1 (with $\Phi$ as above), then $f$ is also positive definite in Sz.-Nagy's sense. However, this is not the case for general $f$; see [2]. As a final comment in relation to Definition 2.1 let us consider the simplest of cases, namely when $X=\mathrm{C}$, in which case also $X^{\prime} \simeq$ C. If $\Phi^{+}:=\left\{z \in X^{\prime}: \operatorname{Re}(z) \geq 0, \operatorname{Im}(z)=0\right\}$, 
then a function $f: S \rightarrow \mathrm{C}$ is $\Phi^{+}$-positive definite if and only if it is positive definite in the classical sense. However, other choices of $\Phi$ are also possible within the scope of Definition 2.1. For example, fix any $w \in C \backslash[0, \infty)$ and define $\Phi_{w}:=\{\gamma w: \gamma \geq 0\} \subseteq X^{\prime}$. Then it is routine to check that a function $f: S \rightarrow C$ is $\Phi_{w}$-positive definite if and only if $\bar{w} f$ is $\Phi^{+}$-positive definite. This illustrates that the "positive definiteness" of a function $f: S \rightarrow X$ is very much dependent on the set of functionals $\Phi \subseteq X^{\prime}$.

Let $\alpha: S \rightarrow[0, \infty)$ be an absolute value. Let $\mathscr{D}_{S}$ denote the linear span of $\{\hat{s}: s \in S\}$ in $C^{S^{*}}$ and let

$$
\mathscr{D}_{S}^{(\alpha)}:=\left\{\left.g\right|_{\alpha}: g \in \mathscr{D}_{S}\right\}
$$

where $\left.g\right|_{\alpha}$ denotes the restriction of $g$ to $S^{\alpha}$. Of course, both $\mathscr{D}_{S}$ and $\mathscr{D}_{S}^{(\alpha)}$ are also algebras under pointwise multiplication. It will always be assumed that $\mathscr{D}_{S}^{(\alpha)}$ is equipped with the supremum norm inherited from the Banach space $C\left(S^{\alpha}\right)$.

LEMMA 2.2. Let $S$ be a unital, commutative semigroup with an involution and $\alpha: S \rightarrow[0, \infty)$ be an absolute value. Let $X$ be a lcHs and $f: S \rightarrow X$ be a $\Phi$-positive definite function which is $\Phi$-scalarly $\alpha$-bounded, that is, each C-valued function $\langle f, \varphi\rangle$, for $\varphi \in \Phi$, is $\alpha$-bounded. If $\Phi$ is a total set (i.e. separates the points of $X)$, then $\Lambda_{f}: \mathscr{D}_{S}^{(\alpha)} \rightarrow X$ specified by

$$
\Lambda_{f}\left(\left.\sum_{j=1}^{n} \beta_{j} \hat{s}_{j}\right|_{\alpha}\right)=\sum_{j=1}^{n} \beta_{j} f\left(s_{j}\right)
$$

is a well defined linear map.

Proof. Suppose that $\left.\sum_{j=1}^{n} \beta_{j} \hat{s}_{j}\right|_{\alpha}=\left.\sum_{k=1}^{m} \gamma_{k} \hat{t}_{k}\right|_{\alpha}$, where $\beta_{j}, \gamma_{k} \in \mathrm{C}$ and $s_{j}, t_{k} \in S$. Fix $\varphi \in \Phi$. Since the scalar function $\langle f, \varphi\rangle$ is $\alpha$-bounded and positive definite, the classical Berg-Maserick theorem guarantees that there exists a unique regular measure $\mu_{\varphi} \geq 0$ with support in $S^{\alpha}$ such that

$$
\langle f(s), \varphi\rangle=\int_{S^{\alpha}} \hat{s} d \mu_{\varphi}, \quad s \in S .
$$

Since $\sum_{j=1}^{n} \beta_{j} \hat{s}_{j}$ and $\sum_{k=1}^{m} \gamma_{k} \hat{t}_{k}$ agree on $S^{\alpha}$, it follows easily from the previous formula that $\left\langle\sum_{j=1}^{n} \beta_{j} f\left(s_{j}\right), \varphi\right\rangle=\left\langle\sum_{k=1}^{m} \gamma_{k} f\left(t_{k}\right), \varphi\right\rangle$. Since $\Phi$ is total we deduce that $\sum_{j=1}^{n} \beta_{j} f\left(s_{j}\right)=\sum_{k=1}^{m} \gamma_{k} f\left(t_{k}\right)$ and hence, that $\Lambda_{f}$ is well defined. The linearity of $\Lambda_{f}$ is routine to verify.

REMARK 2.3. (a) Without the requirement that $f$ is $\Phi$-positive definite the "map" $\Lambda_{f}$ in (1) may fail to be well defined, even when $X=C$ ! The difficulty 
lies with the fact that $\left\{\left.\hat{s}\right|_{\alpha}: s \in S\right\}$ may not be a linearly independent subset of $\mathscr{D}_{S}^{(\alpha)}$; see Remark 6.9.(1) on p. 133 and Proposition 6.1.8 in [4].

(b) An examination of the proof of Lemma 2.2 shows that in order for $\Lambda_{f}$ to be well defined it actually suffices for $\Phi$ to separate the points of the closed subspace $X_{[f]}$ of $X$ generated by the range $f(S):=\{f(s): s \in S\}$ of $f$. This will turn out to be an important point in Section 3. Of course, $X_{[f]}$ is always equipped with the relative topology from $X$. Actually, a close examination of the various proofs shows that in some cases it even suffices for $\Phi$ to separate the points of the linear span of $f(S)$.

There are also other conditions which imply that $\Lambda_{f}$ is well defined. For instance, if $\left\{\left.\hat{s}\right|_{\alpha}: s \in S\right\}$ happens to be a linearly independent subset of $\mathscr{D}_{S}^{(\alpha)}$, which is often the case, then $\Lambda_{f}$ is well defined irrespective of any separating properties of $\Phi$. There may be still other reasons, particular to the special features of $f$ or $\Phi$ or $X$ (or all of them) in a given situation, which also imply that $\Lambda_{f}$ is well defined; see the proof of Proposition 2.17, for instance. We note that the results of this paper, although all formulated in terms of $\Phi$ separating the points of $X_{[f]}$, remain valid whenever this (sufficient) condition is replaced with any other property which ensures that $\Lambda_{f}$ is well defined.

(c) The condition that $\Phi$ is total in $X_{[f]}$ is satisfied in many cases. We note that a large class of spaces $X$ with a natural set $\Phi \subseteq X^{\prime}$ for which $\Phi$ is total for $X$ and such that the positive cone $C_{\Phi}$ is non-trivial (i.e. $C_{\Phi} \neq\{0\}$ ) is the Banach lattices (over R or C). For, in this case $X^{\prime}$ is an order complete Banach lattice, [35, Proposition II.5.5]. Hence, if $\Phi \subseteq X^{\prime}$ is the family of all positive functionals, then certainly $\Phi$ separates the points of $X$ [35, p. 137, Corollary]. Moreover, $C_{\Phi}=X_{+}$is then the cone of positive elements in $X$.

In view of Remark 2.3(a) we will only consider the notion of $\alpha$-boundedness for vector-valued functions which are also $\Phi$-positive definite. This is no restriction since the Berg-Maserick theorem (even in the scalar setting) requires the function to have both of these properties anyway.

Definition 2.4. Let $S$ be a unital, commutative semigroup with an involution and $\alpha: S \rightarrow[0, \infty)$ be an absolute value. Let $X$ be a lcHs and $f: S \rightarrow X$ be a function. Suppose that $\Lambda_{f}$ is well defined and that $f$ is $\Phi$-positive definite. Then $f$ is called $\alpha$-bounded if it is $\Phi$-scalarly $\alpha$-bounded and the linear operator $\Lambda_{f}: \mathscr{D}_{S}^{(\alpha)} \rightarrow X$ given by (1) is weakly compact (i.e. $\Lambda_{f}$ maps the closed unit ball of the normed space $\mathscr{D}_{S}^{(\alpha)}$ into a relatively weakly compact subset of $X$ ).

At a first glance, Definition 2.4 may not seem like a particularly natural extension from the case of scalar-valued functions to vector-valued functions. However, the basic idea should be that $\alpha$-boundedness and $\Phi$-positive definiteness together "somehow" imply that $f$ has a suitable integral representation. 
The following particular example (hopefully) illustrates that Definition 2.4 is indeed quite natural.

Let $X$ be a Banach lattice. To simplify the discussion we assume that $X$ is defined over $\mathrm{R}$, although a similar line of argument applies to the complex case. Let $\Phi \subseteq X^{\prime}$ be the family of all positive functionals, in which case $\Phi$ separates the points of $X$ as $X^{\prime}=\Phi-\Phi$. Accordingly, $\Lambda_{f}$ is certainly well defined; see Lemma 2.2. Suppose that a function $f: S \rightarrow X$ is $\Phi$-positive definite and $\Phi$-scalarly $\alpha$-bounded. By the classical Berg-Maserick theorem applied to $\langle f, \varphi\rangle$, for each $\varphi \in \Phi$, there exists a unique regular, finite Borel measure $\mu_{\varphi} \geq 0$ supported in $S^{\alpha}$ such that

$$
\langle f(s), \varphi\rangle=\int_{S^{\alpha}} \hat{s} d \mu_{\varphi}, \quad s \in S .
$$

Let $M_{r}\left(S^{\alpha}\right)$ denote the space of all regular, R-valued Borel measures on $S^{\alpha}$ equipped with the total variation norm. Then $M_{r}\left(S^{\alpha}\right)$ is also a Banach lattice; it is the dual Banach lattice of $C_{\mathrm{R}}\left(S^{\alpha}\right)$. The uniqueness of $\mu_{\varphi}$, for each $\varphi \in \Phi$, implies that the map $T: \Phi \rightarrow M_{r}\left(S^{\alpha}\right)$ defined by $\varphi \mapsto \mu_{\varphi}$ is additive (i.e. $T\left(\varphi_{1}+\varphi_{2}\right)=T\left(\varphi_{1}\right)+T\left(\varphi_{2}\right)$ for all $\left.\varphi_{j} \in \Phi\right)$. By the extension theorem of L. V. Kantorovic, [1, p. 7], for example, $T$ extends uniquely to a positive linear operator from the Banach lattice $X^{\prime}$ into $M_{r}\left(S^{\alpha}\right)$. By a classical result for Banach lattices it follows that $T$ is automatically continuous, [1, p. 175]. Moreover, for this particular setting, it turns out that $\Lambda_{f}: \mathscr{D}_{S}^{(\alpha)} \rightarrow X$ as given by (1) is necessarily continuous (c.f. Proposition 2.13(i) below); its continuous linear extension to $C_{\mathrm{R}}\left(S^{\alpha}\right)$, possible by virtue of the Stone-Weierstrass theorem which implies that $\mathscr{D}_{S}^{(\alpha)}$ is dense in $C_{\mathrm{R}}\left(S^{\alpha}\right)$, [4, p. 95], is again denoted by $\Lambda_{f}$. It follows from (2) that

$$
\left\langle\Lambda_{f}(g), \varphi\right\rangle=\langle g, T(\varphi)\rangle, \quad g \in \mathscr{D}_{S}^{(\alpha)}, \quad \varphi \in \Phi,
$$

and hence, also for all $\varphi \in X^{\prime}$ as $X^{\prime}=\Phi-\Phi$. Accordingly, $T=\Lambda_{f}^{\prime}$ is the dual operator of $\Lambda_{f}: C_{\mathrm{R}}\left(S^{\alpha}\right) \rightarrow X$. For each fixed set $E \in \mathscr{B}\left(S^{\alpha}\right)$ define a linear map $m(E): X^{\prime} \rightarrow \mathrm{R}$ by $x^{\prime} \mapsto T\left(x^{\prime}\right)(E)$. Then the inequality

$$
\left|T\left(x^{\prime}\right)(E)\right| \leq\left\|T\left(x^{\prime}\right)\right\| \leq\|T\| .\left\|x^{\prime}\right\|, \quad x^{\prime} \in X^{\prime},
$$

where $\left\|T\left(x^{\prime}\right)\right\|$ denotes the total variation of the measure $T\left(x^{\prime}\right)$, shows that $m(E) \in X^{\prime \prime}$. The finitely additive set function $m: \mathscr{B}\left(S^{\alpha}\right) \rightarrow X^{\prime \prime}$ so defined has the property that $\left\langle x^{\prime}, m\right\rangle: E \mapsto\left\langle x^{\prime}, m(E)\right\rangle$ is regular and $\sigma$-additive for all $x^{\prime} \in X^{\prime}$, has range $m\left(\mathscr{B}\left(S^{\alpha}\right)\right):=\left\{m(E): E \in \mathscr{B}\left(S^{\alpha}\right)\right\}$ a bounded subset of $M_{r}\left(S^{\alpha}\right)$ and satisfies

$$
\left\langle f(s), x^{\prime}\right\rangle=\int_{S^{\alpha}} \hat{s} d\left\langle x^{\prime}, m\right\rangle, \quad s \in S,
$$


for each $x^{\prime} \in X^{\prime}$. The crucial point is to ensure that $m$ actually assumes its values in $X$ rather than in $X^{\prime \prime}$, that is, to ensure for each $E \in \mathscr{B}\left(S^{\alpha}\right)$ that the norm continuous linear functional $m(E): X^{\prime} \rightarrow \mathrm{R}$ is actually weak-* continuous on $X^{\prime}$. Because $\Lambda_{f}^{\prime}=T$, by Gantmacher's theorem, [1, p. 284], this is equivalent with $\Lambda_{f}$ being a weakly compact operator, which is precisely the condition required in Definition 2.4. This example, although somewhat special, hopefully gives some insight as to why Definition 2.4 is "reasonable".

Getting back to Definition 2.4 in general, some further comments are in order. Note that $\Lambda_{f}: \mathscr{D}_{S}^{(\alpha)} \rightarrow X$ is weakly compact if and only if it is weakly compact when also interpreted as being $X_{[f]}$-valued. This follows from the fact that $X_{[f]}$ is a weakly closed subset of $X$ and that the natural injection of $X_{[f]}$ into $X$, being obviously continuous is also weakly continuous, [34, p. 158]. So, it is irrelevant whether we consider $\Lambda_{f}$ as being $X$-valued or $X_{[f]^{-}}$ valued. Moreover, whenever $\alpha(s) \neq 0$ for each $s \in S$ (which is no restriction in practice) the function $\frac{f}{\alpha}$ necessarily has relatively weakly compact range. This is a simple consequence of the weak compactness of the mapping $\Lambda_{f}$ and the fact that $\frac{\hat{s}}{\alpha(s)}$ is in the unit ball of $\mathscr{D}_{S}^{(\alpha)}$ for each $s \in S$.

Still considering Definition 2.4, let $q$ be a continuous seminorm on $X$. Since $\Lambda_{f}$ is weakly compact, the image of the unit ball in $\mathscr{D}_{S}^{(\alpha)}$ is relatively weakly compact in $X$ and hence, is a bounded subset of $X$. That is, there is $M_{q}>0$ such that $q\left(\Lambda_{f}(g)\right) \leq M_{q}$ for all $g \in \mathscr{D}_{S}^{(\alpha)}$ with $\|g\|_{\infty} \leq 1$. If follows that

$$
q\left(\Lambda_{f}(g)\right) \leq M_{q}\|g\|_{\infty}, \quad g \in \mathscr{D}_{S}^{(\alpha)} .
$$

Accordingly, $\Lambda_{f}$ is necessarily continuous. Suppose that the lcHs $X_{[f]}$ is sequentially complete. Since $\mathscr{D}_{S}^{(\alpha)}$ is sequentially dense in $C\left(S^{\alpha}\right)$, it follows that $\Lambda_{f}$ has a unique extension to a continuous linear map from $C\left(S^{\alpha}\right)$ into $X_{[f]}$ and hence, also into $X$ (even though $X$ may not be sequentially complete). This extension, again denoted by $\Lambda_{f}$, is also weakly compact.

Let $X$ be a lcHs. A $\sigma$-additive map $m: \Sigma \rightarrow X$, where $\Sigma$ is a $\sigma$-algebra of sets, is called a vector measure. The Orlicz-Pettis theorem [18, p. 4] implies that $m$ is $\sigma$-additive if and only if the C-valued set function $\left\langle m, x^{\prime}\right\rangle$ defined by $E \mapsto\left\langle m(E), x^{\prime}\right\rangle$, for $E \in \Sigma$, is $\sigma$-additive for each $x^{\prime} \in X^{\prime}$. If $\Sigma$ is the $\sigma$-algebra of Borel sets of some compact Hausdorff space, then $m$ is called regular if each complex measure $\left\langle m, x^{\prime}\right\rangle$, for $x^{\prime} \in X^{\prime}$, is regular, [17, p. 4]. The

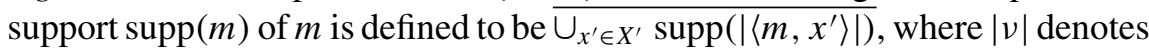
the total variation measure of a complex measure $v$ on $\Sigma$. For the definition of a C-valued, $\Sigma$-measurable function being $m$-integrable with respect to a vector measure $m: \Sigma \rightarrow X$ we refer to [19, Chapter II]. In particular, if $X^{[m]}$ is sequentially complete, then every bounded $\Sigma$-measurable function is necessarily $m$-integrable, $\left[19\right.$, p. 26]. Here $X^{[m]}$ denotes the closed subspace 
of $X$ generated by the range $m(\Sigma):=\{m(E): E \in \Sigma\}$ of $m$, and equipped with the relative topology from $X$.

Definition 2.5. A vector measure $m: \Sigma \rightarrow X$ is called $\Phi$-positive if its range $m(\Sigma)$ is contained in the positive cone $C_{\Phi}$, that is, if and only if, $\langle m, \varphi\rangle$ is a non-negative measure on $\Sigma$ for each $\varphi \in \Phi$.

In the case when $X$ is a Banach space this agrees with the definition given in [12, p. 606].

A similar comment as made after Definition 2.1 is also relevant to Definition 2.5. Namely, the "positiveness" of a given vector measure is very much dependent on the set of functionals $\Phi \subseteq X^{\prime}$. Moreover, there exist measures which are not $\Phi$-positive for any non-trivial $\Phi$, already in the simplest case when $X=\mathrm{C}$. To see this let $\Sigma:=\mathscr{B}(\mathrm{C})$ and define $m: \Sigma \rightarrow X$ by $m:=\delta_{1}-\delta_{-1}$, where $\delta_{w}$ denotes the Dirac point mass at $w \in \mathrm{C}$. Then the only functional $\varphi \in X^{\prime} \simeq \mathrm{C}$ for which $\langle m, \varphi\rangle \geq 0$ on $\Sigma$ is $\varphi=0$ and so there is no non-trivial set $\Phi \subseteq X^{\prime}$ for which $m$ is $\Phi$-positive. Since $C \simeq S^{*}$ (for the semigroup $S=\mathrm{N}_{0} \times \mathrm{N}_{0}$ defined in the Example in Section 3) and $m$ is regular and compactly supported, we note that the generalized Laplace transform $\hat{m}: S \rightarrow X$ (see (3) below) can be $\Phi$-scalarly $\alpha$-bounded for some absolute value $\alpha$ on $S$, but fail to be $\Phi$-positive definite for every non-trivial set $\Phi \subseteq X^{\prime}$

Recall that a lcHs is called quasicomplete if each closed, bounded subset is complete.

Proposition 2.6. Let X be a sequentially complete lcHs. Let $S$ be a unital, commutative semigroup with an involution, $m: \mathscr{B}\left(S^{*}\right) \rightarrow X$ be a regular, compactly suppported vector measure and $\hat{m}: S \rightarrow X$ be the generalized Laplace transform of $m$, that is,

$$
\hat{m}(s):=\int_{S^{*}} \hat{s} d m, \quad s \in S .
$$

Let $\alpha: S \rightarrow[0, \infty)$ be the absolute value

$$
\alpha(s):=\sup \{|\hat{s}(\rho)|: \rho \in \operatorname{supp}(m)\}, \quad s \in S .
$$

Suppose that $\Phi \subseteq X^{\prime}$ separates the points of $X^{[m]}$, that $X^{[m]}$ is quasicomplete and that $m$ is $\Phi$-positive. Then $\hat{m}$ takes its values in $X^{[m]}$ and $\hat{m}$ is both $\Phi$ positive definite and $\alpha$-bounded.

Proof. It is routine to check that $\alpha$ is an absolute value and that $\operatorname{supp}(m) \subseteq$ $S^{\alpha}$. Since $\hat{s}$ is continuous on the compact set $K:=\operatorname{supp}(m)$ it follows that $\hat{s}$ is bounded and Borel measurable on $K$, for each $s \in S$. According to earlier 
remarks, since $m$ can be interpreted as being $X^{[m]}$-valued, it follows that the vector integrals (3) are well defined elements of $X^{[m]}$. A direct calculation yields

$$
\left\langle\sum_{j, k=1}^{n} c_{j} \bar{c}_{k} \hat{m}\left(s_{j} s_{k}^{-}\right), x^{\prime}\right\rangle=\int_{K}\left|\sum_{j=1}^{n} c_{j} \hat{s}_{j}\right|^{2} d\left\langle m, x^{\prime}\right\rangle, \quad x^{\prime} \in X^{\prime},
$$

for all choices of finitely many elements $\left\{c_{1}, \ldots, c_{n}\right\} \subseteq \mathrm{C}$ and $\left\{s_{1}, \ldots, s_{n}\right\} \subseteq S$, where we have used the general formula $\left\langle\int_{K} g d m, x^{\prime}\right\rangle=\int_{K} g d\left\langle m, x^{\prime}\right\rangle$ valid for all $x^{\prime} \in X^{\prime}$ and all $m$-integrable functions $g$, [19, p. 21]. So, if $\varphi \in \Phi$, then $\langle m, \varphi\rangle \geq 0$ and we see that $\hat{m}$ is $\Phi$-positive definite.

Fix $\varphi \in \Phi$. Then it follows from (3) that $\langle\hat{m}(s), \varphi\rangle=\int_{K} \hat{s} d\langle m, \varphi\rangle$ and hence, since $\langle m, \varphi\rangle \geq 0$, that

$$
\begin{aligned}
|\langle\hat{m}(s), \varphi\rangle| \leq \int_{K}|\hat{s}| & d\langle m, \varphi\rangle \\
\leq & \langle m(K), \varphi\rangle \sup \{|\hat{s}(\rho)|: \rho \in K\}=\langle m(K), \varphi\rangle \cdot \alpha(s)
\end{aligned}
$$

for each $s \in S$. This shows that $\hat{m}$ is $\Phi$-scalarly $\alpha$-bounded.

A direct calculation via (1) and (3) establishes that

$$
\Lambda_{\hat{m}}\left(\left.\sum_{j=1}^{n} \beta_{j} \hat{s}_{j}\right|_{\alpha}\right)=\int_{K}\left(\sum_{j=1}^{n} \beta_{j} \hat{s}_{j}\right) d m .
$$

Since all integrals of the form $\int_{K} g d m$ with $\|g\|_{\infty} \leq 1$ ( on $S^{\alpha}$ hence also on $K$ ) belong to the balanced convex hull in $X^{[m]}$ of the range of $m$, $[19$, p. 75 , Lemma 1], and the range of $m$ is a relatively weakly compact set in $X^{[m]}$, [39], it follows from the quasicompleteness of $X^{[m]}$, that the closed balanced convex hull of the range of $m$ is weakly compact in $X^{[m]}$, [34, p. 189], [39]. Accordingly, $\Lambda_{\hat{m}}$ is a weakly compact map from $\mathscr{D}_{S}^{(\alpha)}$ into $X^{[m]}$. That is, $\hat{m}$ is $\alpha$-bounded.

It is the following converse of Proposition 2.6 which can be interpreted as a vector-valued analogue of the classical Berg-Maserick theorem.

Proposition 2.7. Let $S$ be a unital, commutative semigroup with an involution and $\alpha: S \rightarrow[0, \infty)$ be an absolute value. Let $X$ be a lcHs and $f: S \rightarrow X$ be a function. Suppose that $X_{[f]}$ is quasicomplete and $\Phi \subseteq X^{\prime}$ separates the points of $X_{[f]}$. If $f$ is $\alpha$-bounded and $\Phi$-positive definite, then there exists a unique regular vector measure $m: \mathscr{B}\left(S^{\alpha}\right) \rightarrow X$ which takes its values in $X_{[f]}$, is $\Phi$-positive and satisfies

$$
f(s)=\int_{S^{\alpha}} \hat{s} d m, \quad s \in S .
$$


Proof. By hypothesis the linear map $\Lambda_{f}: C\left(S^{\alpha}\right) \rightarrow X_{[f]}$, which is the unique extension of (1) from $\mathscr{D}_{S}^{(\alpha)}$ to $C\left(S^{\alpha}\right)$, is weakly compact. By the vectorvalued Riesz representation theorem, [18, Proposition 1], there exists a unique regular vector measure $m: \mathscr{B}\left(S^{\alpha}\right) \rightarrow X_{[f]}$ such that

$$
\Lambda_{f}(g)=\int_{S^{\alpha}} g d m, \quad g \in C\left(S^{\alpha}\right) .
$$

Since (1) implies that $\Lambda_{f}\left(\left.\hat{s}\right|_{\alpha}\right)=f(s)$, the formula (5) follows.

Let $\varphi \in \Phi$. Then $\langle f, \varphi\rangle$ is positive definite and $\alpha$-bounded and so there is a unique regular measure $\mu_{\varphi} \geq 0$ with support in $S^{\alpha}$ such that $\langle f(s), \varphi\rangle=$ $\int_{S^{\alpha}} \hat{s} d \mu_{\varphi}$ for each $s \in S$. But, (5) implies that also $\langle f(s), \varphi\rangle=\int_{S^{\alpha}} \hat{s} d\langle m, \varphi\rangle$, for all $s \in S$, with $\langle m, \varphi\rangle$ regular and supported in $S^{\alpha}$. Accordingly,

$$
\int_{S^{\alpha}} g d\langle m, \varphi\rangle=\int_{S^{\alpha}} g d \mu_{\varphi}, \quad g \in \mathscr{D}_{S}^{(\alpha)} .
$$

Then the density of $\mathscr{D}_{S}^{(\alpha)}$ in $C\left(S^{\alpha}\right)$ and an approximation argument via the dominated convergence theorem implies that (6) is valid for all $g \in C\left(S^{\alpha}\right)$. Consequently, the classical Riesz representation theorem ensures that $\langle m, \varphi\rangle=$ $\mu_{\varphi}$ as measures. In particular, $\langle m, \varphi\rangle \geq 0$. Since $\varphi \in \Phi$ is arbitrary it follows that $m$ is $\Phi$-positive.

Given a $\Phi$-positive definite and $\Phi$-scalarly $\alpha$-bounded function $f: S \rightarrow X$, with $\Phi$ a total set of functionals for $X_{[f]}$, we say that $f$ is $\alpha$-dominated if there exists a finite regular measure $\mu: \mathscr{B}\left(S^{\alpha}\right) \rightarrow[0, \infty)$ such that

$$
\left\|\Lambda_{f}(g)\right\| \leq \int_{S^{\alpha}}|g| d \mu, \quad g \in \mathscr{D}_{S}^{(\alpha)} .
$$

Suppose now that $X_{[f]}$ is a Banach space, in which case the unique continuous extension of $\Lambda_{f}$ from $\mathscr{D}_{S}^{(\alpha)}$ to $C\left(S^{\alpha}\right)$ still fulfills (7). It is known that there exists a unique regular vector measure $m: \mathscr{B}\left(S^{\alpha}\right) \rightarrow X_{[f]}$ of finite variation (see [7, Ch. 1] for the definition) such that

$$
\Lambda_{f}(g)=\int_{S^{\alpha}} g d m, \quad g \in C\left(S^{\alpha}\right) .
$$

Indeed, this follows from [8, p. 380] and the fact that (7) actually holds for all $g \in C\left(S^{\alpha}\right)$. An argument as in the proof of Proposition 2.6 then shows that $\Lambda_{f}$ is also weakly compact. Accordingly, if $f$ is $\alpha$-dominated, then it is also $\alpha$-bounded. So, we have the following more specialized version of Proposition 2.7. 
Proposition 2.8. Let $S$ be a unital, commutative semigroup with an involution and $\alpha: S \rightarrow[0, \infty)$ be an absolute value. Let $X$ be a lcHs and $f: S \rightarrow X$ be a function such that $X_{[f]}$ is a Banach space. If $\Phi \subseteq X^{\prime}$ is total for $X_{[f]}$ and $f$ is $\Phi$-positive definite and $\alpha$-dominated, then there exists a unique regular vector measure $m: \mathscr{B}\left(S^{\alpha}\right) \rightarrow X_{[f]}$ of finite variation which is $\Phi$-positive and satisfies (5).

Remark 2.9. Let $X$ be a lcHs and $m: \mathscr{B}\left(S^{*}\right) \rightarrow X$ be a regular, compactly supported vector measure such that $X^{[m]}$ is a Banach space and $m$ has finite variation when interpreted as being $X^{[m]}$-valued. If $\Phi \subseteq X^{\prime}$ is total for $X^{[m]}$ and $m$ is $\Phi$-positive, then the generalized Laplace transform $\hat{m}: S \rightarrow X$ as given by (3) takes its values in $X^{[m]}$, is $\Phi$-positive definite and is $\alpha$-dominated for the absolute value

$$
\alpha(s):=\sup \{|\hat{s}(\rho)|: \rho \in \operatorname{supp}(m)\}, \quad s \in S .
$$

Indeed, that $\hat{m}$ takes its values in $X^{[m]}$, is $\Phi$-positive definite and $\Phi$-scalarly $\alpha$ bounded follows as for Proposition 2.6. Let $|m|$ denote the variation measure of $m$. Then it follows from (4) that

$$
\left\|\Lambda_{\hat{m}}(g)\right\|=\left\|\int_{S^{\alpha}} g d m\right\| \leq \int_{S^{\alpha}}|g| d|m|, \quad g \in \mathscr{D}_{S}^{(\alpha)},
$$

where the stated inequality is a basic fact for vector measures of finite variation, [8, Ch. II, §8]. Accordingly, (7) is satisfied, that is, $\hat{m}$ is $\alpha$-dominated.

The $\alpha$-boundedness of a vector-valued function is not always easy to verify in practice. We now present a criterion in this direction which will be useful in the next section.

Let $X$ be a lcHs. A set $\Phi \subseteq X^{\prime}$ is called full if for every continuous seminorm $q$ on $X$ there exists a seminorm $p: X^{\prime} \rightarrow[0, \infty)$ such that

$$
q(x) \leq \sup \left\{|\langle x, \varphi\rangle| / p(\varphi): \varphi \in \Phi \backslash p^{-1}(\{0\})\right\}<\infty, \quad x \in X .
$$

If $X$ is a Banach space, then this is satisfied (for instance) whenever there exists a constant $M>0$ such that

$$
\|x\| \leq M \sup \left\{|\langle x, \varphi\rangle| /\|\varphi\|_{X^{\prime}}: \varphi \in \Phi \backslash\{0\}\right\}<\infty, \quad x \in X .
$$

The inequality (9) is taken as a definition of full in [12]. Moreover, examples in [12, Section 5] show that this condition is often satisfied. It is clear from (8) that any full family of functionals $\Phi \subseteq X^{\prime}$ is also total for $X$.

Proposition 2.10. Let $S$ be a unital, commutative semigroup with an involution and $\alpha: S \rightarrow[0, \infty)$ be an absolute value. Let $X$ be a lcHs and 
$f: S \rightarrow X$ be a function. Suppose that $\Phi \subseteq X^{\prime}$ is a full space of functionals for $X_{[f]}$ and that $f$ is $\Phi$-positive definite.

(i) If $f$ is $\Phi$-scalarly $\alpha$-bounded, then the linear map $\Lambda_{f}: \mathscr{D}_{S}^{(\alpha)} \rightarrow X$ as given by (1) is continuous.

(ii) Suppose that $X_{[f]}$ is a Banach space which does not contain an isomorphic copy of the sequence space $c_{0}$. If $f$ is $\Phi$-scalarly $\alpha$-bounded, then $f$ is $\alpha$-bounded.

Proof. (i) For each $\varphi \in \Phi$ the classical Berg-Maserick theorem guarantees a unique regular measure $\mu_{\varphi} \geq 0$ supported in $S^{\alpha}$ such that

$$
\langle f(s), \varphi\rangle=\int_{S^{\alpha}} \hat{s} d \mu_{\varphi}, \quad s \in S .
$$

It is routine to verify that

$$
\left\langle\Lambda_{f}(g), \varphi\right\rangle=\int_{S^{\alpha}} g d \mu_{\varphi}, \quad g \in \mathscr{D}_{S}^{(\alpha)} .
$$

Given a continuous seminorm $q$ on $X_{[f]}$ let $p:\left(X_{[f]}\right)^{\prime} \rightarrow[0, \infty)$ be any seminorm satisfying (8). Then

$$
\begin{aligned}
q\left(\Lambda_{f}(g)\right) & \leq \sup \left\{\left|\int_{S^{\alpha}} g d \mu_{\varphi}\right| / p(\varphi): \varphi \in \Phi \backslash p^{-1}(\{0\})\right\} \\
& \leq\|g\|_{\infty} \sup \left\{\mu_{\varphi}\left(S^{\alpha}\right) / p(\varphi): \varphi \in \Phi \backslash p^{-1}(\{0\})\right\} \\
& =\|g\|_{\infty} \sup \left\{\langle f(e), \varphi\rangle / p(\varphi): \varphi \in \Phi \backslash p^{-1}(\{0\})\right\} .
\end{aligned}
$$

Hence, with $M_{q}:=\sup \left\{\langle f(e), \varphi\rangle / p(\varphi): \varphi \in \Phi \backslash p^{-1}(\{0\})\right\}<\infty$ (see (8)) we have

$$
q\left(\Lambda_{f}(g)\right) \leq M_{q}\|g\|_{\infty}, \quad g \in \mathscr{D}_{S}^{(\alpha)} .
$$

This shows that $\Lambda_{f}$ is continuous.

Part (ii) follows from (i) and a result of A. Pelczyński which states that every continuous linear map from $C(K)$, where $K$ is any compact Hausdorff space, into a Banach space not containing a copy of $c_{0}$ is necessarily weakly compact; see Theorem 15 on p. 159 of [7] and [7, p. 180].

It is worth noting that all weakly sequentially complete Banach spaces (hence, all reflexive ones) cannot contain a copy of $c_{0}$.

Remark 2.11. For the case of $X_{[f]}$ a Banach space and $\Phi \subseteq X^{\prime}$ a full set of functionals for $X_{[f]}$ satisfying (9), the calculation in the proof of part (i) of 
Proposition 2.10 which leads to (10) yields the inequality

$$
\|f(s)\| \leq M\|f(e)\| \alpha(s), \quad s \in S,
$$

where we have substituted $g=\left.\hat{s}\right|_{\alpha}$ and used the estimate $\left\|\left.\hat{s}\right|_{\alpha}\right\|_{\infty}=\sup \{|\rho(s)|$ : $\left.\rho \in S^{\alpha}\right\} \leq \alpha(s)$. This is a vector analogue of the well known inequality

$$
|h(s)| \leq h(e) \alpha(s), \quad s \in S,
$$

valid for any scalar-valued positive definite function $h: S \rightarrow C$ which is $\alpha$-bounded, [4, p. 90, Proposition 1.12].

The following result illustrates that there is an important class of Banach spaces in which there always exists a natural family of functionals $\Phi \subseteq X^{\prime}$ which is full and such that $C_{\Phi}$ is non-trivial.

Proposition 2.12. Let $X$ be a Banach lattice (over $\mathrm{R}$ or $\mathrm{C}$ ) and let $\Phi \subseteq X^{\prime}$ be the cone of all positive functionals. Then $\Phi$ is full.

PROof. For a standard reference to Banach lattices we refer to [35], for example. Suppose first that $X$ is a real Banach lattice. For each $x \in X$, we have $\|x\|=\sup \left\{\left|\left\langle x, x^{\prime}\right\rangle\right|: x^{\prime} \in X^{\prime},\left\|x^{\prime}\right\| \leq 1\right\}$. So, choose any $x^{\prime}$ satisfying $\left\|x^{\prime}\right\| \leq 1$. Then $x^{\prime}=\left(x^{\prime}\right)^{+}-\left(x^{\prime}\right)^{-}$with both $\left(x^{\prime}\right)^{+},\left(x^{\prime}\right)^{-} \in \Phi$, and so

$$
\left|\left\langle x, x^{\prime}\right\rangle\right| \leq\left|\left\langle x,\left(x^{\prime}\right)^{+}\right\rangle\right|+\left|\left\langle x,\left(x^{\prime}\right)^{-}\right\rangle\right| .
$$

But, $0 \leq\left(x^{\prime}\right)^{+} \leq\left|x^{\prime}\right|$ and so $\left\|\left(x^{\prime}\right)^{+}\right\| \leq\left\|\left|x^{\prime}\right|\right\|=\left\|x^{\prime}\right\| \leq 1$. Similarly $\left\|\left(x^{\prime}\right)^{-}\right\| \leq 1$ and it follows that

$$
\left|\left\langle x, x^{\prime}\right\rangle\right| \leq 2 \sup \{|\langle x, \varphi\rangle|: \varphi \in \Phi,\|\varphi\| \leq 1\} .
$$

Since this is valid for every $x^{\prime}$ satisfying $\left\|x^{\prime}\right\| \leq 1$ we have

$$
\|x\| \leq 2 \sup \{|\langle x, \varphi\rangle| /\|\varphi\|: \varphi \in \Phi \backslash\{0\}\}, \quad x \in X .
$$

Accordingly, $\Phi$ is a full family of functionals.

Suppose that $X$ is a complex Banach lattice. Then $X$ is the complexification of a real Banach lattice $Y$ and the norm in $X$ is defined by

$$
\|x\|:=\||x|\|, \quad x \in X,
$$

where $|x|$ is a suitably defined element of the positive cone of $Y$, [35, Chapter II, $\S 11]$. The conclusion then follows from the definition of positive functionals in a complex Banach lattice [35, p. 135] combined with the result for real Banach lattices. 
Combining Propositions 2.7, 2.10 and 2.12 yields the following useful result.

Proposition 2.13. Let $S$ be a unital, commutative semigroup with an involution and $\alpha: S \rightarrow[0, \infty)$ be an absolute value. Let $X$ be a Banach lattice and $\Phi \subseteq X^{\prime}$ be the cone of all positive functionals. Let $f: S \rightarrow X$ be a function which is $\Phi$-positive definite and $\Phi$-scalarly $\alpha$-bounded. Then,

(i) the linear map $\Lambda_{f}: \mathscr{D}_{S}^{(\alpha)} \rightarrow X$ is continuous.

Suppose, in addition, that $X$ does not contain a copy of $c_{0}$. Then,

(ii) the linear map $\Lambda_{f}: \mathscr{D}_{S}^{(\alpha)} \rightarrow X$ is weakly compact and hence, $f$ is necessarily $\alpha$-bounded. In particular, there exists a unique regular, $\Phi$ positive vector measure $m: \mathscr{B}\left(S^{\alpha}\right) \rightarrow X$ such that

$$
f(s)=\int_{S^{\alpha}} \hat{s} d m, \quad s \in S .
$$

There is a partial converse to Proposition 2.12 which shows that the existence of a full set of functionals $\Phi$ for a Banach space $X$ implies some rather strong order properties on $X$. For ease of presentation we again suppose that $X$ is a real Banach space.

So, suppose that $\Phi \subseteq X^{\prime}$ is a full set of functionals, that is, (9) is satisfied for some constant $M>0$. It is routine to check that $C_{\Phi}$ satisfies both $C_{\Phi}+C_{\Phi} \subseteq$ $C_{\Phi}$ and $\beta C_{\Phi} \subseteq C_{\Phi}$, for all $\beta \geq 0$, and that $C_{\Phi}$ is weakly (hence, also norm) closed in $X$. Accordingly, $X$ is an ordered Banach space in the sense of [34, p. 222]. Moreover, it is clear from (9) that the formula

$$
\||\|\mid\|:=\sup \{|\langle x, \varphi\rangle| /\|\varphi\|: \varphi \in \Phi \backslash\{0\}\}, \quad x \in X,
$$

defines an equivalent norm on $X$ which satisfies $\||x\||\leq\|\mid x+y\|$ for all $x, y \in C_{\Phi}$. In particular, this inequality implies that $\|y\||\leq\|\mid\| x \|$ whenever $x, y \in C_{\Phi}$ satisfy $0 \leq y \leq x$, and that $C_{\Phi}$ is a normal cone in $(X,\|\cdot\| \|)$, [34, p. 215]. Since $\Phi$ clearly separates the points of $X$ we see that $C_{\Phi}$ is also a proper cone, i.e. $\left(-C_{\Phi}\right) \cap C_{\Phi}=\{0\}$. The normality of $C_{\Phi}$ implies that the dual cone $\hat{\Phi} \subseteq X^{\prime}$ of $C_{\Phi}$, defined by

$$
\hat{\Phi}:=\left\{x^{\prime} \in X^{\prime}:\left\langle x, x^{\prime}\right\rangle \geq 0 \text { for all } x \in C_{\Phi}\right\},
$$

satisfies $\Phi \subseteq \hat{\Phi}$ (obviously) and $X^{\prime}=\hat{\Phi}-\hat{\Phi}$, [34, p. 220]. Moreover, $\hat{\Phi}$ is a strict $\mathfrak{B}$-cone, [34, p. 221] which is easily verified to be norm closed in $X^{\prime}$. Accordingly, $X^{\prime}$ is also an ordered Banach space, with respect to the cone $\hat{\Phi},[34$, p. 222$]$, and has the property that every positive linear functional 
$\psi: X^{\prime} \rightarrow$ R (i.e. $\left.\psi(\hat{\Phi}) \subseteq[0, \infty)\right)$ is necessarily norm continuous, [34, p. 228]. The following result summarizes the previous discussion.

Proposition 2.14. Let $X$ be a (real) Banach space and $\Phi \subseteq X^{\prime}$ be a full space of functionals. Then (11) defines an equivalent norm in $X$ with respect to which

$$
C_{\Phi}:=\{x \in X:\langle x, \varphi\rangle \geq 0 \text { for all } \varphi \in \Phi\}
$$

is a (closed) normal cone and $X$ is an ordered Banach space. Moreover, $\|y\| \leq$ $\||x|||$ whenever $x, y \in C_{\Phi}$ satisfy $0 \leq y \leq x$.

The dual cone $\hat{\Phi} \subseteq X^{\prime}$ given by

$$
\hat{\Phi}:=\left\{x^{\prime} \in X^{\prime}:\left\langle x, x^{\prime}\right\rangle \geq 0 \text { for all } x \in C_{\Phi}\right\}
$$

contains $\Phi$, is a norm closed, strict $\mathfrak{B}$-cone satisfying $X^{\prime}=\hat{\Phi}-\hat{\Phi}$, and makes $X^{\prime}$ an ordered Banach space with the property that every R-valued, positive linear functional on $X^{\prime}$ is automatically norm continuous on $X^{\prime}$.

Now for an application. On semigroups "without" involution (i.e. the identity function is taken to be the involution) there is an interesting subclass of the positive definite functions, namely the completely monotone functions, [4, Ch. $4, \S 6]$. In the scalar case (i.e. for R-valued functions) these turn out to be mixtures of $[0,1]$-valued characters. So, let us formulate a vector analogue.

Let $X$ be a real lcHs, $\Phi \subseteq X^{\prime}$ be a non-empty subset and $C_{\Phi} \subseteq X$ be the corresponding positive cone. Let $S$ be a unital, commutative semigroup "without" involution. For each $t \in S$ define $E_{t}: X^{S} \rightarrow X^{S}$ by

$$
E_{t} f: s \mapsto f(s+t), \quad s \in S,
$$

for each $f \in X^{S}$, and $\nabla_{t}: X^{S} \rightarrow X^{S}$ by

$$
\nabla_{t} f: s \mapsto f(s)-f(s+t), \quad s \in S,
$$

for each $f \in X^{S}$. Note that $\nabla_{t}=I-E_{t}$. Since $\left\{E_{t}: t \in S\right\}$ is a commuting family of operators on $X^{S}$, so is $\left\{\nabla_{t}: t \in S\right\}$. A function $f: S \rightarrow X$ is called $\Phi$-completely monotone if,

(i) $f(S) \subseteq C_{\Phi}$, and

(ii) $\left(\nabla_{t_{1}} \nabla_{t_{2}} \cdots \nabla_{t_{n}} f\right)(S) \subseteq C_{\Phi}$ for every finite set $\left\{t_{j}\right\}_{j=1}^{n} \subseteq S$.

This is equivalent with each scalar function $\langle f, \varphi\rangle: S \rightarrow \mathrm{R}$, for $\varphi \in \Phi$, being completely monotone. In particular, $\langle f, \varphi\rangle$ is bounded for each $\varphi \in \Phi,[4$, p. 130]. Let $\hat{S}:=\left\{\rho \in S^{*}:|\rho(s)| \leq 1\right.$ for all $\left.s \in S\right\}$. Then $\hat{S}$ is a compact subsemigroup of $S^{*},[4$, p. 96], and $\hat{S}$ is the set of all bounded characters on $S$. A famous result, originally due to G. Choquet, states that any (R-valued) completely monotone function on $S$ is the generalized Laplace transform of 
some (unique) Radon measure $\mu \geq 0$, concentrated on the set $\hat{S}_{+}$of nonnegative bounded characters on $S$ (which are then obviously [0,1]-valued), [4, Theorem 4.6.4]. So, $\langle f, \varphi\rangle=\hat{\mu}_{\varphi}$ with $\mu_{\varphi} \in M_{+}\left(\hat{S}_{+}\right)$, for each $\varphi \in \Phi$.

Proposition 2.15. Let $S$ be a unital, commutative semigroup without involution, $X$ be a real lcHs and $f: S \rightarrow X$ be a function. Suppose that $X_{[f]}$ is quasicomplete and $\Phi \subseteq X^{\prime}$ separates the points of $X_{[f]}$. Let $f$ be $\Phi$-completely monotone and 11 -bounded, where 11 is the absolute value which constantly takes the value 1 on $S$. Then there exists a unique regular, $\Phi$-positive vector measure $m: \mathscr{B}\left(\hat{S}_{+}\right) \rightarrow X$ with values in $X_{[f]}$ such that

$$
f(s)=\int_{\hat{S}_{+}} \hat{s} d m, \quad s \in S .
$$

Moreover, the range $f(S)$ of $f$ is a relatively weakly compact subset of $X_{[f]}$.

Proof. As noted above, $\langle f, \varphi\rangle=\hat{\mu}_{\varphi}$ for some unique $\mu_{\varphi} \in M_{+}\left(\hat{S}_{+}\right)$. In particular, $\langle f, \varphi\rangle$ is positive definite and so $f$ is $\Phi$-positive definite as $\varphi \in \Phi$ is arbitrary. Note that $S^{\mathbb{1}}=\hat{S}$. By Proposition 2.7 there is a unique regular, $\Phi$-positive vector measure $m: \mathscr{B}(\hat{S}) \rightarrow X_{[f]}$ such that $f=\hat{m}$. We need to check that $\operatorname{supp}(m) \subseteq \hat{S}_{+}$. Let $A \subseteq \hat{S} \backslash \hat{S}_{+}$be measurable. Then

$$
\langle m(A), \varphi\rangle=\mu_{\varphi}(A)=0, \quad \varphi \in \Phi,
$$

(argue as in the proof of Proposition 2.7) and so $m(A)=0$ as $\Phi$ separates the points of $X_{[f]}$. It follows that $\operatorname{supp}(m) \subseteq \hat{S}_{+}$.

Since $0 \leq \hat{s} \leq \mathbb{1}$, for each $\hat{s}$ restricted to $\hat{S}_{+}$and each $s \in S$, it follows that

$$
f(S) \subseteq\left\{\int_{\hat{S}_{+}} g d m: g \text { measurable and } 0 \leq g \leq \mathbb{1}\right\} .
$$

Accordingly, $f(S)$ is relatively weakly compact; see the proof of Proposition 2.6.

We point out that this is the only result in the paper where the totality of $\Phi$ is genuinely needed, i.e. other than as a sufficient condition ensuring that $\Lambda_{f}$ is well defined.

The following consequence of Proposition 2.15 is of interest in its own right.

Corollary 2.16. Let $X$ be a real Banach space not containing a copy of $c_{0}$ and $\Phi \subseteq X^{\prime}$ be a full set of functionals. Given any $\Phi$-completely monotone function $f: S \rightarrow X$, where $S$ is a unital, commutative semigroup 
"without" involution, there exists a unique regular, Ф-positive vector measure $m: \mathscr{B}\left(\hat{S}_{+}\right) \rightarrow X$ such that

$$
f(s)=\int_{\hat{S}_{+}} \hat{s} d m, \quad s \in S .
$$

Proof. An examination of the proof of Proposition 2.15 shows that $f$ is both $\Phi$-positive definite and $\Phi$-scalarly 11 -bounded. By Proposition 2.10(ii) the function $f$ is necessarily 1 -bounded. The conclusion then follows from Proposition 2.15.

We note that the assumption on $\Phi$ in the above Corollary is always fulfilled when $X$ is a real Banach lattice and $\Phi$ is taken to be the positive cone of $X^{\prime}$; see Proposition 2.12.

We end this section with a further application of the above results to certain kinds of stochastic processes. As is traditional in this subject, we will write the semigroup operation additively. The well developed theory of weakly stationary random processes is concerned with stochastic processes having finite second moments whose time parameter varies in an abelian group $G$. Such a process can be succinctly described as a Hilbert space valued map $X: G \rightarrow H$, where $H=L^{2}(\mu)$ for some probability measure $\mu$ for which the expectation (= correlation in case $X$ is centered, which is no restriction) $E[X(g) \overline{X(k)}]=\langle X(g), X(k)\rangle$ depends only on $g-k$. It is well known (and has been widely used) that such a process admits an integral representation in the form $X(g)=\int_{\hat{G}} \hat{g} d m$ for some unique, regular measure $m: \mathscr{B}(\hat{G}) \rightarrow H$ (defined on the dual group $\hat{G}$ ) which is orthogonally scattered, i.e. $m(E) \perp m(F)$ whenever $E, F \in \mathscr{B}(\hat{G})$ are disjoint. In many instances the time parameter may only vary in a commutative semigroup $S$ with an involution. In this case we call a function $f: S \rightarrow H$ additively correlated if $\langle f(s), f(t)\rangle$ is purely a function of $s+t^{-}$. For the case of the identity function as involution and with $S=\mathrm{N}_{0}$ this terminology was introduced in [25]. A natural question is to decide under which additional conditions such a function $f$ possesses an integral representation similar to the one just mentioned for abelian groups. This was answered in [29, Theorem 1] for the case when $f$ is bounded and the involution is the identity function. The following proposition can be interpreted as a natural extension of this result.

Proposition 2.17. Let $S$ be a unital, commutative semigroup with an involution and $\alpha: S \rightarrow[0, \infty)$ be an absolute value. Let $H$ be a (complex) Hilbert space and $f: S \rightarrow H$ be an additively correlated function such that $\|f(\cdot)\|$ is $\alpha$-bounded as a scalar function on $S$. Then there exists a unique 
regular, orthogonally scattered measure $m: \mathscr{B}\left(S^{\alpha}\right) \rightarrow H$ such that

$$
f(s)=\int_{S^{\alpha}} \hat{s} d m, \quad s \in S .
$$

Proof. By hypothesis there exists a function $\psi: S \rightarrow \mathrm{C}$ such that $\langle f(s), f(t)\rangle$ $=\psi\left(s+t^{-}\right)$, for all $s, t \in S$. It follows that

$$
\sum_{j, k=1}^{n} c_{j} \bar{c}_{k} \psi\left(s_{j}+s_{k}^{-}\right)=\left\|\sum_{j=1}^{n} c_{j} f\left(s_{j}\right)\right\|_{H}^{2} \geq 0,
$$

for all choices of finitely many elements $\left\{c_{j}\right\}_{j=1}^{n} \subseteq \mathrm{C}$ and $\left\{s_{j}\right\}_{j=1}^{n} \subseteq S$. Furthermore,

$$
|\psi(s)|=|\langle f(s), f(0)\rangle| \leq\|f(s)\|_{H}\|f(0)\|_{H} \leq C\|f(0)\|_{H} \alpha(s), \quad s \in S,
$$

for some constant $C>0$. This establishes that $\psi$ is $\alpha$-bounded and positive definite. Accordingly, the classical Berg-Maserick theorem guarantees a unique regular measure $\mu \geq 0$ exists, with support in $S^{\alpha}$, such that $\psi=\hat{\mu}$. It follows from (12) and $\psi=\hat{\mu}$ that

$$
\left\|\left.\sum_{j=1}^{n} c_{j} \hat{s}_{j}\right|_{\alpha}\right\|_{L^{2}(\mu)}=\left\|\sum_{j=1}^{n} c_{j} f\left(s_{j}\right)\right\|_{H},
$$

for all choices $\left\{c_{j}\right\}_{j=1}^{n} \subseteq \mathrm{C}$ and $\left\{s_{j}\right\}_{j=1}^{n} \subseteq S$. It is clear from (13) that $\Lambda_{f}$ : $\mathscr{D}_{S}^{(\alpha)} \rightarrow H$ as given by (1) is well defined.

Let $\Phi \subseteq H^{\prime}(\simeq H)$ denote the family of all finite sums of the form $\sum_{j} c_{j} f\left(s_{j}\right)$ for which $\sum_{j} c_{j} \hat{s}_{j} \geq 0$ pointwise $\mu$-a.e. on $S^{\alpha}$; the family $\Phi$ is well defined because of (13). Furthermore, for a fixed element $\sum_{j=1}^{n} c_{j} f\left(s_{j}\right) \in \Phi$, we see that

$$
\begin{aligned}
\left\langle\sum_{\ell, k=1}^{p} d_{\ell} \bar{d}_{k} f\left(t_{\ell}+t_{k}^{-}\right), \sum_{j=1}^{n} c_{j} f\left(s_{j}\right)\right\rangle & =\sum_{j, k, \ell} \bar{c}_{j} d_{\ell} \bar{d}_{k} \psi\left(s_{j}^{-}+t_{\ell}+t_{k}^{-}\right) \\
& =\sum_{j, k, \ell} \bar{c}_{j} d_{\ell} \bar{d}_{k} \int_{S^{\alpha}} \overline{\hat{s}}_{j} \hat{t}_{\ell} \overline{\hat{t}}_{k} d \mu \\
& =\int_{S^{\alpha}} \overline{\left(\sum_{j=1}^{n} c_{j} \hat{s}_{j}\right)} \cdot\left|\sum_{\ell=1}^{p} d_{\ell} \hat{t}_{\ell}\right|^{2} d \mu \\
& =\int_{S^{\alpha}}\left(\sum_{j=1}^{n} c_{j} \hat{s}_{j}\right) \cdot\left|\sum_{\ell=1}^{p} d_{\ell} \hat{t}_{\ell}\right|^{2} d \mu
\end{aligned}
$$


is a non-negative number for all choices of finitely many elements $\left\{d_{\ell}\right\}_{\ell=1}^{p} \subseteq \mathrm{C}$ and $\left\{t_{\ell}\right\}_{\ell=1}^{p} \subseteq S$. Together with the inequality

$$
\left\|\left\langle f(t), \sum_{j=1}^{n} c_{j} f\left(s_{j}\right)\right\rangle \mid \leq C \alpha(t)\right\| \sum_{j=1}^{n} c_{j} f\left(s_{j}\right) \|_{H}, \quad t \in S,
$$

this shows that $f$ is $\Phi$-positive definite and $\Phi$-scalarly $\alpha$-bounded. Moreover, for $\left.\sum_{j=1}^{r} \beta_{j} \hat{s}_{j}\right|_{\alpha} \in \mathscr{D}_{S}^{(\alpha)}$, it follows from (13) and the definition of $\Lambda_{f}$ that

$$
\left\|\Lambda_{f}\left(\left.\sum_{j=1}^{r} \beta_{j} \hat{s}_{j}\right|_{\alpha}\right)\right\|_{H}=\left\|\sum_{j=1}^{r} \beta_{j} \hat{s}_{j}\right\|_{L^{2}(\mu)} \leq \mu\left(S^{\alpha}\right)^{1 / 2}\left\|\left.\sum_{j=1}^{r} \beta_{j} \hat{s}_{j}\right|_{\alpha}\right\|_{\infty},
$$

i.e. $\Lambda_{f}: \mathscr{D}_{S}^{(\alpha)} \rightarrow H$ is continuous with $\left\|\Lambda_{f}\right\| \leq \mu\left(S^{\alpha}\right)^{1 / 2}=\|f(0)\|_{H}$. In particular, $\Lambda_{f}$ is weakly compact (as $H$ is reflexive) and we see that $\Lambda_{f}$ is $\alpha$-bounded.

Proposition 2.7 and Remark 2.3(b) now imply the existence of a unique regular, $\Phi$-positive vector measure $m: \mathscr{B}\left(S^{\alpha}\right) \rightarrow H_{[f]}$ satisfying

$$
f(s)=\int_{S^{\alpha}} \hat{s} d m, \quad s \in S .
$$

The isometric linear map $\left.\sum_{j} c_{j} f\left(s_{j}\right) \mapsto \sum_{j} c_{j} \hat{s}_{j}\right|_{\alpha}$, from the linear span of $f(S) \subseteq H_{[f]}$ onto $\mathscr{D}_{S}^{(\alpha)}$ (considered as a subspace of $L^{2}(\mu)$ ), extends uniquely to an isometric isomorphism $J$ of $H_{[f]}$ onto $L^{2}(\mu)$. Moreover, for any $s \in S$, we have that

$$
\int_{S^{\alpha}} \hat{s} d(J \circ m)=J\left(\int_{S^{\alpha}} \hat{s} d m\right)=J(f(s))=\left.\hat{s}\right|_{\alpha} .
$$

But, if $n: \mathscr{B}\left(S^{\alpha}\right) \rightarrow L^{2}(\mu)$ is the canonical vector measure given by $n(E)=$ $\chi_{E}$, for each $E \in \mathscr{B}\left(S^{\alpha}\right)$, then $n$ is regular and also satisfies

$$
\int_{S^{\alpha}} \hat{s} d n=\left.\hat{s}\right|_{\alpha}, \quad s \in S .
$$

Hence, $\int_{S^{\alpha}} g d(J \circ m)=\int_{S^{\alpha}} g d n$ for all $g \in \mathscr{D}_{S}^{(\alpha)}$. Then a simple approximation argument (using the density of $\mathscr{D}_{S}^{(\alpha)}$ in $C\left(S^{\alpha}\right)$ and the dominated convergence theorem for vector measures) shows that $\int_{S^{\alpha}} g d(J \circ m)=\int_{S^{\alpha}} g d n$ for all $g \in C\left(S^{\alpha}\right)$. Since both $n$ and $J \circ m$ are regular, the uniqueness part of the vector valued Riesz representation theorem implies that $n=J \circ m$. Since $n$ is clearly orthogonally scattered and $J$ is isometric, it follows from the polarization identity that $m$ is also orthogonally scattered. 


\section{Representation of $*$-semigroups of scalar operators}

The aim of this section is to apply the results of $\S 1$ to deduce an integral formula for representations of certain kinds of semigroups of scalar operators in a Banach space. In the Hilbert space setting there is a vast literature on this topic, where the Mackey-Wermer theorem implies that scalar operators reduce essentially to normal operators. For Banach spaces the known results are not so well developed. Often there are restrictions on the Banach space, such as reflexivity or weak sequential completeness. In other cases the underlying semigroup $S$ is either of a rather particular nature (e.g. $S$ is a group or $S=\mathrm{N}_{0}$ or $S=\mathrm{R}_{+}$etc.) or $S$ is required to be imbedded in an appropriate topological group and the associated operator semigroup should have some continuity properties. As a sample of results in this direction we refer to [6], [14], [15], [16], [20], [21], [22], [24], [26], [27], [28], [31], [32], [36], [37] and the references therein, for example.

The kind of restrictions indicated above lie in the very nature of things; general Banach spaces simply do not have the rich geometric structure available in Hilbert spaces. The methods of positive definite functions compensate for this to some extent because of the rather rich order structure imposed on the Banach space. Furthermore, there are virtually no restrictions on the underlying semigroup $S$ when applying such methods. Of course, there must be some additional properties placed on the operator semigroup involved. Such properties, roughly speaking, compensate for the fact that selfadjoint and normal operators have no analogue in the Banach space setting and hence, the powerful methods of $C^{*}$-algebras are not available.

In order to motivate and formulate a suitable notion of a $*$-representation for semigroups of scalar operators, we begin with a consideration of generalized Laplace transforms of multiplicative, operator-valued measures.

Throughout this section $Y$ denotes a (complex) Banach space. The vector space of all continuous linear operators of $Y$ into itself is denoted by $L(Y)$. To stress that $L(Y)$ is equipped with the strong operator topology (i.e. the topology of pointwise convergence on $Y$ ) we will write $L_{s}(Y)$. It is an easy consequence of the Banach-Steinhaus theorem that $L_{s}(Y)$ is a quasicomplete lcHs; its topology is determined by the family of seminorms $\left\{q_{y}: y \in Y\right\}$, where

$$
q_{y}: T \mapsto\|T y\|, \quad T \in L_{s}(Y) .
$$

All continuous linear functionals $\xi$ on $L_{s}(Y)$ have the form

$$
\xi: T \mapsto \sum_{j=1}^{n}\left\langle T y_{j}, y_{j}^{\prime}\right\rangle, \quad T \in L_{s}(Y),
$$


for some finite family of elements $\left\{y_{j}\right\}_{j=1}^{n} \subseteq Y$ and $\left\{y_{j}^{\prime}\right\}_{j=1}^{n} \subseteq Y^{\prime}$, [34, p. 139].

A vector measure $P: \Sigma \rightarrow L_{s}(Y)$, where $\Sigma$ is a $\sigma$-algebra of subsets of some non-empty set $\Omega$, is usually called an operator-valued measure. From the definition of the topology in $L_{s}(Y)$ this means that the $Y$-valued function $P y$ defined by $P y: E \mapsto P(E) y$ is $\sigma$-additive on $\Sigma$, for each $y \in Y$. In view of (15) and the Orlicz-Pettis theorem, this is equivalent to the $\mathrm{C}$-valued function $\left\langle P y, y^{\prime}\right\rangle: E \mapsto\left\langle P(E) y, y^{\prime}\right\rangle$ being $\sigma$-additive on $\Sigma$ for each $y \in Y$ and $y^{\prime} \in Y^{\prime}$.

An operator-valued measure $P: \Sigma \rightarrow L_{s}(Y)$ which is multiplicative on $\Sigma$ (i.e. $P(E \cap F)=P(E) P(F)$ for all $E, F \in \Sigma$ ) and satisfies $P(\Omega)=I$ (the identity operator on $Y$ ) is called a spectral measure. For such measures it turns out that the only $P$-integrable functions $h: \Omega \rightarrow C$ are those which are bounded and $\Sigma$-measurable, [11, XVIII Theorem 2.11(c)]. In this case, if we define $P(h):=\int_{\Omega} h d P$, then for each $E \in \Sigma$ the operator $\int_{E} h d P:=P(h) P(E)=P(E) P(h)$ is the unique element of $L_{s}(Y)$ which satisfies $\left\langle\int_{E} h d P, \xi\right\rangle=\int_{E} h d\langle P, \xi\rangle$ for all $\xi \in\left(L_{s}(Y)\right)^{\prime}$. A set $E \in \Sigma$ is called $P$-null whenever $P(E)=0$. The multiplicativity of $P$ implies that $P(F)=0$ for all sets $F \in \Sigma$ satisfying $F \subseteq E$. Two $P$-integrable functions are called $P$-equivalent if they only differ on a $P$-null set. Then $L^{1}(P)$ denotes the usual space of equivalence classes of $P$-integrable functions modulo $P$-equivalence. An operator $T \in L(Y)$ is called a scalar-type spectral operator (briefly, a scalar operator) if there exists some spectral measure $P: \Sigma \rightarrow L_{s}(Y)$ and $h \in L^{1}(P)$ such that $T=P(h),[11, \mathrm{XV}, \S 4$ and XVII, $\S 2]$.

Let $P: \Sigma \rightarrow L_{S}(Y)$ be a spectral measure. Then the integration map $I_{P}: L^{1}(P) \rightarrow L_{s}(Y)$ defined by $h \mapsto P(h)$ is injective. For each $y \in Y$ define a seminorm $q_{y}(P)$ on $L^{1}(P)$ by

$$
q_{y}(P): h \mapsto q_{y}(P(h)), \quad h \in L^{1}(P),
$$

where $q_{y}$ is given by (14). The family of seminorms $\left\{q_{y}(P): y \in Y\right\}$ determines a lcH-topology $\tau(P)$ on $L^{1}(P)$ and $I_{P}$ becomes a bicontinuous linear and algebraic isomorphism of $\left(L^{1}(P), \tau(P)\right)$ onto its range $\mathscr{R}\left(I_{P}\right)$, equipped with the relative topology from $L_{s}(Y)$. If the range $P(\Sigma)$ of $P$ is a closed subset of $L_{s}(Y)$, which is always the case if $Y$ is separable for instance, then the lcHs $L^{1}(P)$ is $\tau(P)$-complete. In particular, the range $\mathscr{R}\left(I_{P}\right)$ is then a complete subspace of the quasicomplete lcHs $L_{s}(Y)$. Moreover, $\mathscr{R}\left(I_{P}\right)$ coincides with the closed subalgebra $\mathscr{A}_{P}$ of $L_{s}(Y)$ generated by $P(\Sigma)$. The algebra $\mathscr{A}_{P}$ has an involution $*$, namely $P(h)^{*}:=P(\bar{h})$ for each $h \in L^{1}(P)$. Each element of the dual space $\mathscr{A}_{P}^{\prime}$ has the special form (c.f. (15))

$$
T \mapsto\left\langle T y, y^{\prime}\right\rangle, \quad T \in \mathscr{A}_{P},
$$


for some $y \in Y$ and $y^{\prime} \in Y^{\prime}$, [13]. Functionals of the form (16) will be denoted by $y \otimes y^{\prime}$.

Define $\|\cdot \mid\|: Y \rightarrow[0, \infty)$ by

$$
\|y\|:=\sup \left\{\|P(f) y\|: f \in L^{1}(P), 0 \leq|f| \leq 1\right\}, \quad y \in Y .
$$

Then \|\|$\cdot \|$ is an equivalent norm on $Y$ with the property that $\||P(h) y \||=$ $\||| P(|h|) y \mid\|$ for all $y \in Y$ and $h \in L^{1}(P)$. In particular, if $\||P(h) \||$ denotes the operator norm calculated with respect to the norm $\||\cdot|||$ in $Y$, then

$$
\left\|\left|P ( h ) \left\|\left|=\left\||P(|h|) \||, \quad h \in L^{1}(P) .\right.\right.\right.\right.\right.
$$

All of the above notions and facts about the integration map and spectral measures can be found in [9], [10], [11], for example.

Let $P: \Sigma \rightarrow L_{s}(Y)$ be a spectral measure and fix a vector $y \in Y$. An element $y^{\prime} \in Y^{\prime}$ is called a Bade functional for $y$ with respect to $P$ if

(i) $\left\langle P(E) y, y^{\prime}\right\rangle \geq 0$ for each $E \in \Sigma$, and

(ii) $P(E) y=0$ whenever $E \in \Sigma$ satisfies $\left\langle P(E) y, y^{\prime}\right\rangle=0$.

The existence of Bade functionals follows from [3, Theorem 3.1], after noting that $P(\Sigma)$ is a $\sigma$-complete Boolean algebra of projections; see the proof of [11, XVII Corollary 3.10]. In particular, the $Y$-valued vector measure $P y$ and the non-negative scalar measure $\left\langle P y, y^{\prime}\right\rangle$ have the same null sets whenever $y^{\prime}$ is a Bade functional for $y$ with respect to $P$.

Given $y \in Y$, let $P(\Sigma)[y]$ denote the cyclic space generated by $y$, that is, the closed subspace of $Y$ generated by $\{P(E) y: E \in \Sigma\}$. In the notation of Section 1 we have $P(\Sigma)[y]=Y^{[P y]}$. We note that the restrictions to the invariant subspace $P(\Sigma)[y]$ of all members of $P(\Sigma)$ forms a $\sigma$-complete Boolean algebra of projections in $P(\Sigma)$ [y], [11, XVII Corollary 3.11], and that $y$ is a cyclic vector for this family of restrictions (in the Banach space $P(\Sigma)[y]$ ). So, if we choose any fixed Bade functional $y^{\prime} \in Y^{\prime}$ for $y$ with respect to $P$, then the linear span of $\Phi_{y}:=\left\{P(F)^{\prime} y^{\prime}: F \in \Sigma\right\}$ is a weak-* dense subspace of $(P(\Sigma)[y])^{\prime},\left[11\right.$, XVII Lemma 3.13]. Define $\Phi:=\cup_{y \in Y}\left\{y \otimes \xi: \xi \in \Phi_{y}\right\}$, where $y \otimes \xi$ is given by (16). Then $\Phi \subseteq \mathscr{A}_{P}^{\prime}$ and the properties of Bade functionals ensure that the spectral measure $P$ is $\Phi$-positive. Moreover, $\Phi$ separates the points of $\mathscr{A}_{P}$. To see this, let $T=P(h)$ for some $h \in L^{1}(P)$ and suppose that $\langle T, \zeta\rangle=0$ for all $\zeta \in \Phi$. Fix $y \in Y$. Then $\left\langle\left(\int_{\Omega} h d P\right) y, \xi\right\rangle=$ 0 for all $\xi \in \Phi_{y}$. Approximating $h$ uniformly by $\Sigma$-simple functions and using the dominated convergence theorem for vector measures, $[19$, p. 30 , Theorem 2], applied to $P y: \Sigma \rightarrow P(\Sigma)[y]$, it follows that $\left(\int_{\Omega} h d P\right) y=$ $\int_{\Omega} h d(P y)$ is an element of $P(\Sigma)[y]$. Since the linear span of the restrictions $\left.\Phi_{y}\right|_{P(\Sigma)[y]}$ is weak-* dense in $(P(\Sigma)[y])^{\prime}$ and $\left\langle\int_{\Omega} h d(P y), \xi\right\rangle=0$ for all 
$\xi \in \Phi_{y}$ it follows that $\left(\int_{\Omega} h d P\right) y=0$. But, $y \in Y$ is arbitrary and so $T=\int_{\Omega} h d P=0$ as an element of $\mathscr{A}_{P} \subseteq L(Y)$.

Let $S$ be a unital, commutative semigroup with an involution and $P$ : $\mathscr{B}\left(S^{*}\right) \rightarrow L_{s}(Y)$ be a regular, compactly supported spectral measure with range a closed subset of $L_{s}(Y)$. Let $X$ denote the complete lcHs $\mathscr{A}_{P}:=$ $\mathscr{R}\left(I_{P}\right) \subseteq L_{s}(Y)$ in which case $P$ can be considered as an $X$-valued measure. As noted above $\Phi:=\cup_{y \in Y}\left\{y \otimes \xi: \xi \in \Phi_{y}\right\} \subseteq X^{\prime}$ is a total set of functionals for $X$ and $P$ is $\Phi$-positive. It follows from properties of Bade functionals that

$$
\left\{\int_{\Omega} h d P: h \in L^{1}(P), h \geq 0\right\} \subseteq C_{\Phi} .
$$

Define a map $\mathscr{U}_{P}: S \rightarrow X \subseteq L_{s}(Y)$ by

$$
\mathscr{U}_{P}(s):=\int_{S^{*}} \hat{s} d P, \quad s \in S .
$$

Then $\left\{\mathscr{U}_{P}(s)\right\}_{s \in S}$ is a commutative semigroup of scalar operators, that is, $\mathscr{U}_{P}(s t)=\mathscr{U}_{P}(s) \mathscr{U}_{P}(t)$ for all $s, t \in S$. Moreover, $\mathscr{U}_{P}(e)=I$ and $\mathscr{U}_{P}\left(s^{-}\right)=$ $\mathscr{U}_{P}(s)^{*}:=\int_{S^{*}} \overline{\hat{s}} d P$, for each $s \in S$. With respect to the equivalent norm $\||\cdot|\|$ in $Y$ given by (17) we have $\left\|\mathscr{U}_{P}(s)^{*}\right\|\|=\| \mathscr{U}_{P}(s) \|$ for all $s \in S$; see (18). Moreover, Proposition 2.6 applied to $m:=P$ and $X:=\mathscr{A}_{P}$ implies that $\mathscr{U}_{P}$ is $\Phi$-positive definite and $\alpha$-bounded with respect to the absolute value

$$
\alpha(s):=\sup \{|\hat{s}(\rho)|: \rho \in \operatorname{supp}(P)\}, \quad s \in S .
$$

The following result summarizes the above discussion.

Proposition 3.1. Let $S$ be a unital, commutative semigroup with an involution. Let $Y$ be a Banach space and $P: \mathscr{B}\left(S^{*}\right) \rightarrow L_{s}(Y)$ be a regular, compactly supported spectral measure with range a closed subset of $L_{s}(Y)$. Then there exists a unital, abelian and complete subalgebra $\mathscr{A}_{P} \subseteq L_{S}(Y)$ with an involution $*$, and a total set of functionals $\Phi \subseteq \mathscr{A}_{P}^{\prime}$ for $\mathscr{A}_{P}$ such that $\mathscr{U}_{P}: S \rightarrow L_{s}(Y)$, as defined by (19), is a semigroup of scalar operators which takes its values in $\mathscr{A}_{P}$ and satisfies

(i) $\mathscr{U}_{P}(e)=I$ and $\mathscr{U}_{P}(s t)=\mathscr{U}_{P}(s) \mathscr{U}_{P}(t)$, for all $t, s \in S$,

(ii) $\mathscr{U}_{P}\left(s^{-}\right)=\mathscr{U}_{P}(s)^{*}$, for all $s \in S$,

(iii) $\mathscr{U}_{P}$ is $\Phi$-positive definite and $\alpha$-bounded with respect to the absolute value $\alpha: S \rightarrow[0, \infty)$ given by $(20)$, and

(iv) \|\| $\mathscr{U}_{P}(s)\|\|=\left\|\mathscr{U}_{P}(s)^{*}\right\| \mid$ for an equivalent norm $\|\cdot\| \mid \|$ on $Y$, for all $s \in S$. 
Proposition 3.1 suggests what (perhaps) a natural definition of a $*$-representation for a semigroup of scalar operators in a Banach space should be; see Definition 3.5 below. First we need one further notion.

Definition 3.2 ([34], p. 255). Let $Y$ be a Banach space. A unital subalgebra $\mathscr{A} \subseteq L(Y)$ is called a spectral algebra if it is continuously isomorphic (linearly and multiplicatively) to a $C(K)$-space for some compact Hausdorff space $K$.

In every spectral algebra $\mathscr{A} \subseteq L(Y)$ there is an involution given by $T^{*}=$ $\Gamma(\bar{f})$ if $T=\Gamma(f)$, where $\Gamma: C(K) \rightarrow \mathscr{A}$ is an isomorphism as in Definition 3.2. This involution is independent of the isomorphism $\Gamma$ and the space $C(K)$.

For the class of reflexive Banach spaces, $C$. Ionescu Tulcea introduced the notion of a (D)-algebra (in [14]) as a spectral algebra which is closed in the weak operator topology.

Lemma 3.3. Let $Y$ be a Banach space not containing a copy of $c_{0}$ and $\mathscr{A} \subseteq$ $L(Y)$ be a spectral algebra. Then the closure $\overline{\mathscr{A}}^{w}$ of $\mathscr{A}$ in the weak operator topology is a (D)-algebra. In particular, $\overline{\mathscr{A}}^{w}$ is also a spectral algebra and each element of $\overline{\mathscr{A}}^{w}$ (hence, of $\mathscr{A}$ ) is a scalar operator.

Proof. Let $\Gamma: C(K) \rightarrow \mathscr{A} \subseteq L(Y)$ be an isomorphism as in Definition 3.2. A subset $W \subseteq L(Y)$, for an arbitrary Banach space $Y$, is relatively compact for the weak operator topology if and only if $\{T y: T \in W\}$ is a relatively weakly compact subset of $Y$, for each $y \in Y$. This fact, together with a result of A. Pelczyński (see the proof of Proposition 2.10(ii)), implies that $W:=\left\{\Gamma(h):\|h\|_{\infty} \leq 1\right\}$ is relatively compact for the weak operator topology in $L(Y)$. Since $L_{s}(Y)$ is quasicomplete and the weak operator topology is the weak topology for the lcHs $L_{s}(Y)$, it follows from the vector-valued Riesz representation theorem [18] that there exists a unique regular measure $Q: \mathscr{B}(K) \rightarrow L_{s}(Y)$ such that $\Gamma(h)=\int_{K} h d Q$ for all $h \in C(K)$. An examination of the proof of the Riesz representation theorem given in [18] shows that $Q$ takes its values in $\bar{W}^{w} \subseteq \mathscr{\mathscr { A }}^{w}$. Note that $Q(K)=\Gamma(1)=I$. The homomorphism property of $\Gamma$ implies that $\left(\int_{K} g d Q\right) \cdot\left(\int_{K} h d Q\right)=\int_{K} g h d Q$ for all $g, h \in C(K)$ from which it follows that $Q$ is multiplicative on $\mathscr{B}(K)$, [17, Lemma 3]. So, $Q$ is a spectral measure. Hence, its range $Q(\mathscr{B}(K))$ is a $\sigma$-complete Boolean algebra of projections in the sense of W. Bade [11, XVII Corollary 3.10]. Accordingly, the closure $\mathscr{F}$ of $Q(\mathscr{B}(K))$ in $L_{s}(Y)$ is a complete Boolean algebra of projections, [11, XVII Lemma 3.23], which is clearly contained in $\bar{W}^{w} \subseteq \overline{\mathscr{A}}^{w}$. If $\Omega$ denotes the Stone space of the Boolean algebra $\mathscr{F}$, in which case $\Omega$ is a compact (extremely disconnected) Hausdorff space, then there exists a regular spectral measure $P: \mathscr{B}(\Omega) \rightarrow L_{s}(Y)$ with 
range precisely $\mathscr{F}$, [3, p. 349]. In particular, $P$ has closed range in $L_{s}(Y)$ and so $\mathscr{R}\left(I_{P}\right)$ is the closed subalgebra $\mathscr{A}_{P}$ of $L_{s}(Y)$ generated by $P(\mathscr{B}(\Omega))$, that is, by $\mathscr{F}$. Since $\mathscr{F} \subseteq \overline{\mathscr{A}}^{w}$ we have $\mathscr{A}_{P} \subseteq \overline{\mathscr{A}}^{w}$. But, $Q(\mathscr{B}(K)) \subseteq \mathscr{F} \subseteq \mathscr{A}_{P}$ and $\mathscr{A}_{P}$ is closed and convex in $L_{s}(Y)$ hence, is also weak operator topology closed in $L(Y)$. In particular, $\mathscr{A}_{P}$ is complete in $L_{s}(Y)$ (as $P$ has closed range in $\left.L_{S}(Y)\right)$ and so it follows that $\int_{K} g d Q \in \mathscr{A}_{P}$ for all $g \in L^{1}(Q)$. Since $C(K) \subseteq L^{1}(Q)$ it follows that $\Gamma(h)=\int_{K} h d Q \in \mathscr{A}_{P}$ for all $h \in C(K)$, that is, $\mathscr{A}_{\subseteq} \subseteq \mathscr{A}_{P}$. But, $\mathscr{A}_{P}$ is weak operator topology closed and so $\overline{\mathscr{A}}^{w} \subseteq \mathscr{A}_{P}$. This establishes that $\overline{\mathscr{A}}^{w}=\mathscr{A}_{P}$ and so certainly each element of $\overline{\mathscr{A}}^{w}$ is a scalar operator. Finally, $\mathscr{A}_{P}$ is isomorphic to $C(\Omega)$, [11, XVII Lemma 3.9 \& Corollary 3.17], and so $\overline{\mathscr{A}}^{w}$ is a spectral algebra.

In view of Definition 3.2 and Lemma 3.3, the notion of a spectral algebra can be interpreted as an extension to arbitrary Banach spaces of the notion of a $(D)$-algebra in reflexive spaces.

Lemma 3.4. Let $Y$ be a Banach space and $P: \Sigma \rightarrow L_{s}(Y)$ be any spectral measure. Then the closed subalgebra $\mathscr{A}_{P}$ of $L_{s}(Y)$ generated by the range $P(\Sigma)$ of $P$ is a spectral algebra.

Proof. Since $P(\Sigma)$ is a $\sigma$-complete Boolean algebra of projections (see the proof of [11, XVII Corollary 3.10]) its closure $\mathscr{F}$ in $L_{s}(Y)$ is a complete Boolean algebra of projections, [11, XVII Lemma 3.23]. Moreover, $\mathscr{A}_{P}$ is also the closed subalgebra of $L_{s}(Y)$ generated by $\mathscr{F}$. But, this subalgebra coincides with that generated in $L(Y)$ by the operator norm topology, [11, XVII Corollary 3.17], and hence, is isomorphic to a $C(K)$ space, [11, XVII Lemma 3.9].

It follows from Lemma 3.4 that the algebra $\mathscr{A}_{P}$ of Proposition 3.1 is a spectral algebra, and is weak operator topology closed.

Definition 3.5. Let $S$ be a unital, commutative semigroup with an involution, and let $Y$ be a Banach space. A map $\mathscr{U}: S \rightarrow L(Y)$ is called a positive, exponentially bounded $*$-representation (of operators) if

(i) $\mathscr{U}(e)=I$ and $\mathscr{U}(s t)=\mathscr{U}(s) \mathscr{U}(t)$ for all $s, t \in S$,

(ii) there exists a spectral algebra $\mathscr{A} \subseteq L(Y)$ such that $\mathscr{U}(S) \subseteq \mathscr{A}$ and $\mathscr{U}\left(s^{-}\right)=\mathscr{U}(s)^{*}$ for all $s \in S$,

(iii) there is a set of functionals $\Phi \subseteq\left(L_{s}(Y)\right)^{\prime}$ which separates the points of $\overline{\mathscr{A}}^{w}$ and such that $\mathscr{U}$ is $\Phi$-positive definite, and

(iv) there is an absolute value $\alpha: S \mapsto[0, \infty)$ such that $\mathscr{U}$ is $\alpha$-bounded, that is, $\mathscr{U}$ is $\Phi$-scalarly $\alpha$-bounded and the map $\Lambda_{\mathscr{U}}: \mathscr{D}_{S}^{(\alpha)} \rightarrow L(Y)$ is compact for the weak operator topology. 
REMARK 3.6. (a) Let $Y$ be a Hilbert space. A map $\mathscr{U}: S \rightarrow L(Y)$ is called a *-representation $[38, \S 6]$ if $\mathscr{U}(e)=I$ and $\mathscr{U}\left(s t^{-}\right)=\mathscr{U}(s) \mathscr{U}(t)^{*}$ for all $s, t \in$ $S$. Then each $\mathscr{U}(s)$ is a normal operator and there exists a regular selfadjoint spectral measure $P: \mathscr{B}\left(S^{*}\right) \rightarrow L_{s}(Y)$ with compact support such that $\mathscr{U}=\hat{P}$ is the generalized Laplace transform of $P$, [30, Theorem 2]. Proposition 3.1 implies that properties (i), (iii) and (iv) of Definition 3.5 are satisfied and Lemma 3.4 implies property (ii) with $\mathscr{A}$ being the closed subalgebra of $L_{s}(Y)$ generated by $P\left(\mathscr{B}\left(S^{*}\right)\right.$ ). Hence, $\mathscr{U}$ is a positive, exponentially bounded $*-$ representation in the sense of Definition 3.5.

(b) Property (ii) of Definition 3.5 is a substitute in the Banach space setting for the existence (always) of adjoint operators in the Hilbert space setting. Moreover, if $\mathscr{U}$ is a $*$-representation in a Hilbert space $Y$ (see part (a)), then in (ii) of Definition $3.5 \mathscr{A}$ can always be chosen as the $C^{*}$-algebra generated by the $*$-closed, commutative family of operators $\mathscr{U}(S)$. Furthermore, $\{y \otimes y$ : $y \in Y$ \} can always be taken as $\Phi$ in part (iii), in which case the cone $C_{\Phi}$ consists of the positive operators in $L(Y)$. So, condition (iii) is a substitute in the Banach space setting for the above mentioned properties of the functionals $\{y \otimes y: y \in Y\}$ in the Hilbert space setting. Moreover,

$$
\alpha(s):=\|\mathscr{U}(s)\|, \quad s \in S,
$$

is always an absolute value for a $*$-representation $\mathscr{U}: S \rightarrow L(Y)$ in a Hilbert space $Y$ (the property $\left\|\mathscr{U}(s)^{*}\right\|=\|\mathscr{U}(s)\|$ is crucial here) and $\mathscr{U}$ is always $\Phi$-scalarly $\alpha$-bounded with respect to $\Phi=\{y \otimes y: y \in Y\}$; see the proof of Theorem 2 in [30]. Also, $\Lambda_{\mathscr{U}}: \mathscr{D}_{S}^{(\alpha)} \rightarrow L_{S}(Y)$ is then automatically weak operator compact. Indeed, since $Y$ is reflexive, the argument at the beginning of the proof of Lemma 3.3 shows that it suffices to establish the continuity of $\Lambda_{\mathscr{U}}$. By Proposition 2.10(i) it is then enough to show that $\Phi:=\{y \otimes y: y \in Y\}$ is a full space of functionals for $L_{s}(Y)$. But, if ||$\cdot \mid \|$ denotes the dual norm of the Banach space $L(Y)$-equipped with its operator norm topology - then it is known that

$$
\|T\| \leq 2 \sup \{|\langle T, y \otimes y\rangle| /|\|y \otimes y \mid\|: y \in Y\}, \quad T \in L(Y) .
$$

Hence, for fixed $y \in Y \backslash\{0\}$, consider the continuous seminorm $q_{y}$ on $L_{s}(Y)$ as given by (14). Define $p_{y}:\left(L_{s}(Y)\right)^{\prime} \rightarrow[0, \infty)$ to be the norm $\|\cdot\|\|/ 2\| y \|$. Then

$$
q_{y}(T)=\|T y\| \leq \sup \left\{|\langle T, z \otimes z\rangle| / p_{y}(z \otimes z): z \otimes z \in \Phi \backslash\{0\}\right\}<\infty
$$

for each $T \in L_{s}(Y)$, which shows that (8) is satisfied in $X:=L_{s}(Y)$, that is, $\Phi$ is a full space of functionals. So, condition (iv) is a natural analogue in 
the Banach space setting of the fact that every $*$-representation of (necessarily normal) operators in a Hilbert space is $\alpha$-bounded with respect to the particular absolute value (21).

In view of Remark 3.6(b) our final result can be interpreted as a natural extension to the Banach space setting of the integral representation for $*$ semigroups of normal operators in Hilbert spaces as indicated in Remark 3.6(a); see also [23].

Proposition 3.7. Let $S$ be a unital, commutative semigroup with an involution. Let $Y$ be a Banach space and $\mathscr{U}: S \rightarrow L(Y)$ be a positive, exponentially bounded $*$-representation. Then there exists a unique regular, compactly supported spectral measure $P: \mathscr{B}\left(S^{*}\right) \rightarrow L_{s}(Y)$ such that

$$
\mathscr{U}(s)=\int_{S^{*}} \hat{s} d P, \quad s \in S .
$$

In particular, each $\mathscr{U}(s)$, for $s \in S$, is a scalar operator.

Proof. Let $\mathscr{A} \subseteq L_{S}(Y)$ and $\Phi \subseteq \mathscr{A}^{\prime}=\left(\overline{\mathscr{A}}^{w}\right)^{\prime}$ and $\alpha: S \rightarrow[0, \infty)$ be as in Definition 3.5. Since $X:=\overline{\mathscr{A}}^{w}$ is quasicomplete for the strong operator topology (being a closed subspace of the quasicomplete space $L_{s}(Y)$ ), we can apply Proposition 2.7 to the function $f:=\mathscr{U}$ considered as being $X$ valued. Hence, there is a unique regular, $\Phi$-positive operator-valued measure $P: \mathscr{B}\left(S^{\alpha}\right) \rightarrow \overline{\mathscr{A}}^{w} \subseteq L_{s}(Y)$ such that $\mathscr{U}(s)=\int_{S^{\alpha}} \hat{s} d P(s)$ for each $s \in S$. Note that only (iii) and (iv) of Definition 3.5 are needed here. Since $P\left(S^{\alpha}\right)=$ $\mathscr{U}(e)=I$, in order to show that $P$ is a spectral measure it remains to verify that $P$ is multiplicative on $\mathscr{B}\left(S^{\alpha}\right)$. Define $P(h):=\int_{S^{\alpha}} h d P$ for each bounded Borel function $h$ on $S^{\alpha}$. Then, by (i) and (ii) of Definition 3.5, we have that

$$
P(\hat{s} \hat{t})=\int_{S^{\alpha}} \hat{s} \hat{t} d P=\int_{S^{\alpha}}(s t) \hat{d} P=\mathscr{U}(s t)=\mathscr{U}(s) \mathscr{U}(t)=P(\hat{s}) P(\hat{t})
$$

for all $s, t \in S$ and

$$
P(\overline{\hat{s}})=\int_{S^{\alpha}} \overline{\hat{s}} d P=\int_{S^{\alpha}}\left(s^{-}\right) \hat{d} P=\mathscr{U}\left(s^{-}\right)=\mathscr{U}(s)^{*}=P(\hat{s})^{*}
$$

for all $s \in S$. Since integration is a linear operation it follows that

$$
P(h) P(g)=P(h g), \quad h, g \in \mathscr{D}_{S}^{(\alpha)} .
$$

But, $\mathscr{D}_{S}^{(\alpha)}$ is a dense subalgebra of $C\left(S^{\alpha}\right)$ and so by a known result [17, Lemma 3] it follows from (23) that $P$ is indeed multiplicative. 
Some final comments are in order. In view of Definition 3.2, the fact that $\|h\|_{\infty}=\|\bar{h}\|_{\infty}$ for any element $h$ of a $C(K)$-space, and part (ii) of Definition 3.5 it follows if $\mathscr{U}: S \rightarrow L(Y)$ is any positive, exponentially bounded *-representation, then for an equivalent norm ||$\cdot|\||$ on $Y$ we have

$$
\|\mathscr{U}(s)\|=\left\|\mathscr{U}(s)^{*} \mid\right\|, \quad s \in S .
$$

In particular, it is then immediate from (15) that $\mathscr{U}$ is $\Phi$-scalarly $\alpha$-bounded for the particular absolute value given by $\alpha(s):=\|\mathscr{U}(s)\|$ and for every $\Phi$ satisfying (iii) of Definition 3.5. Moreover, if $\overline{\langle\mathscr{U}\rangle_{u}}$ denotes the closed subalgebra of $L(Y)$ generated by $\mathscr{U}(S)$ with respect to the operator norm, then $\overline{\langle\mathscr{U}\rangle_{u}}$ is contained in some spectral algebra which is weak operator topology closed. Indeed, it follows from (22) that $\overline{\langle\mathscr{U}\rangle_{u}}$ is contained in the closed subalgebra $\mathscr{A}_{P}$ of $L_{S}(Y)$ generated by $P\left(\mathscr{B}\left(S^{\alpha}\right)\right)$. The conclusion then follows from Lemma 3.4. In particular, $\overline{\langle\mathscr{U}\rangle_{u}}$ is contained in a $(D)$-algebra. Moreover, $\mathscr{A}_{P} \subseteq \overline{\mathscr{A}}^{w}$. Indeed, as seen in the proof of Lemma 3.3

$$
P\left(\mathscr{B}\left(S^{\alpha}\right)\right) \subseteq \overline{\left\{\Gamma(h):\|h\|_{\infty} \leq 1\right\}^{w}} \subseteq \overline{\mathscr{A}}^{w},
$$

where $\Gamma: C(K) \rightarrow \mathscr{A}$ is an isomorphism as in Definition 3.2, and so also $\mathscr{A}_{P} \subseteq \overline{\mathscr{A}}^{w}$. Since $\Lambda_{\mathscr{U}}\left(\left.\sum_{j=1}^{n} \beta_{j} \hat{s}_{j}\right|_{\alpha}\right)=\int_{S^{\alpha}}\left(\sum_{j=1}^{n} \beta_{j} \hat{s}_{j}\right) d P \in \mathscr{A}_{P}$, we see that the continuous extension of $\Lambda_{\mathscr{U}}$ from $\mathscr{D}_{S}^{(\alpha)}$ to $C\left(S^{\alpha}\right)$ takes its values in $\mathscr{A}_{P} \subseteq \overline{\mathscr{A}}^{w}$ (and is again weakly compact of course). Moreover, the weak operator compactness of $\Lambda_{\mathscr{U}}$ is the same whether we interpret $\Lambda_{\mathscr{U}}$ as being $\overline{\mathscr{A}}^{w}$-valued or $L_{s}(Y)$-valued; see the discussion after Definition 2.4.

We conclude with the following

Example 3.8. Let $D$ be the closure of a bounded, open, connected subset of $\mathrm{C}$, and let $\mu$ denote planar Lebesgue measure on $D$. Fix $p \in[1, \infty)$ and consider $Y:=L^{p}(D, \mu)$, the usual Banach space of $p$-th power $\mu$-integrable functions.

The set $\mathrm{N}_{0}:=\{0,1,2, \cdots\}$ determines a commutative semigroup $S=$ $\mathrm{N}_{0} \times \mathrm{N}_{0}$ via the operation $(m, n) .(u, v)=(m+u, n+v)$, in which case $e=(0,0)$ is the identity element. An involution is defined in $S$ by the formula $(m, n)^{-}=(n, m)$, for each $(m, n) \in S$.

For $z \in \mathrm{C}$, the function $\rho_{z}: S \rightarrow \mathrm{C}$ given by $\rho_{z}(m, n)=z^{m} \bar{z}^{n}$, for each $(m, n) \in S$, is a character for $S$ and all characters are of this form, that is, $S^{*} \simeq$ C (also topologically), [4, pp. 116-117].

For each $\phi \in C(D)$ let $M_{\phi} \in L(Y)$ denote the multiplication operator $M_{\phi}: f \mapsto \phi f$, for $f \in Y$, and define $\mathscr{A}:=\left\{M_{\phi}: \phi \in C(D)\right\}$. Since $\left\|M_{\phi}\right\|=\|\phi\|_{\infty}:=\sup \{|\phi(z)|: z \in D\}$, for each $\phi \in C(D)$, it is clear that $\mathscr{A}$ is a spectral algebra. Moreover, it is known that $\overline{\mathscr{A}}^{w}=\left\{M_{\phi}: \phi \in L^{\infty}(D, \mu)\right\}$. 
Let $\psi(z)=z$, for $z \in D$, and define $\mathscr{U}: S \rightarrow L(Y)$ by

$$
\mathscr{U}((m, n))=\left(M_{\psi}\right)^{m}\left(M_{\bar{\psi}}\right)^{n}, \quad(m, n) \in S .
$$

Then certainly $\mathscr{U}(S) \subseteq \mathscr{A}$ and so (i) and (ii) of Definition 3.5 are satisfied. If $F:=\{f \in Y: f \geq 0\}$ and $G:=\left\{g \in L^{q}(D, \mu): g \geq 0\right\}$, where $q$ is the conjugate index to $p$ (i.e. $p^{-1}+q^{-1}=1$ if $p>1$ and $q=\infty$ if $p=1$ ), then it is routine to verify that $\Phi:=\{f \otimes g: f \in F, g \in G\}$ separates the points of $\overline{\mathscr{A}}^{w}$. Moreover, if $f \otimes g \in \Phi$ then

$$
\langle\mathscr{U}((m, n)), f \otimes g\rangle=\int_{D} z^{m} \bar{z}^{n} f g d \mu, \quad(m, n) \in S .
$$

Define an absolute value $\alpha: S \rightarrow[0, \infty)$ by $\alpha(s):=\|\mathscr{U}(s)\|$, for $s \in S$. Then it can be calculated that $S^{\alpha}=\left\{z \in \mathrm{C}:|z| \leq\|\psi\|_{\infty}\right\}$ and hence, $D \subseteq S^{\alpha}$. It follows from (24) that

$$
|\langle\mathscr{U}((m, n)), f \otimes g\rangle| \leq C\|\psi\|_{\infty}^{m+n}=C \alpha((m, n)), \quad(m, n) \in S,
$$

where $C=\int_{D} f g d \mu$. Accordingly, $\langle\mathscr{U}(\cdot), f \otimes g\rangle$ is $\alpha$-bounded. Since $(f g) d \mu$ is a positive, compactly supported measure on C it follows from (24) and (25) that $\langle\mathscr{U}(\cdot), f \otimes g\rangle$ is also positive definite, [4, p. 117]. This establishes that $\mathscr{U}$ is $\Phi$-positive definite and $\Phi$-scalarly $\alpha$-bounded.

Given $\beta_{j} \in \mathrm{C}$ and $\left(m_{j}, n_{j}\right) \in S$, for $1 \leq j \leq n$, we have that

$$
\Lambda_{\mathscr{U}}\left(\left.\sum_{j=1}^{n} \beta_{j} z^{m_{j}} \bar{z}^{n_{j}}\right|_{\alpha}\right)=M_{\sum_{j=1}^{n} \beta_{j} z^{m_{j}} \bar{z}^{n_{j}}}
$$

and so

$$
\left\|\Lambda_{\mathscr{U}}\left(\left.\sum_{j=1}^{n} \beta_{j} z^{m_{j}} \bar{z}^{n_{j}}\right|_{\alpha}\right)\right\|=\left\|\sum_{j=1}^{n} \beta_{j} z^{m_{j}} \bar{z}^{n_{j}}\right\|_{\infty} \leq \sup _{z \in S^{\alpha}}\left|\sum_{j=1}^{n} \beta_{j} z^{m_{j}} \bar{z}^{n_{j}}\right| .
$$

Hence, $\Lambda_{\mathscr{U}}$ is continuous for the operator norm topology and so is certainly continuous for the weak operator topology. Since $Y$ does not contain a copy of $c_{0}$ (as $Y$ is reflexive if $1<p<\infty$ and weakly sequentially complete if $p=1)$, an argument as in the proof of Lemma 3.3 shows that $\Lambda_{\mathscr{U}}: \mathscr{D}_{S}^{(\alpha)} \rightarrow$ $L(Y)$ is compact for the weak operator topology. Accordingly, $\mathscr{U}$ is a positive, exponentially bounded $*$-representation.

The spectral measure $P: \mathscr{B}\left(S^{\alpha}\right) \rightarrow L_{s}(Y)$ as guaranteed by Proposition 3.7 can be explicitly given. Its support is the subset $D$ of $S^{\alpha}$ and, for each Borel subset $E$ of $S^{\alpha}$, the projection $P(E) \in L(Y)$ is given by $f \mapsto \chi_{E \cap D} f$, for $f \in Y$. 


\section{REFERENCES}

1. Aliprantis, C. D. and Burkinshaw, O., Positive Operators, Academic Press, Orlando, 1985.

2. Arveson, W. B., Subalgebras of $C^{*}$-algebras, Acta. Math. 123 (1969), 141-224.

3. Bade, W. G., On Boolean algebras of projections and algebras of operators, Trans. Amer. Math. Soc. 80 (1955), 345-359.

4. Berg, C., Christensen, J. P. R. and Ressel, P., Harmonic Analysis on Semigroups, Theory of Positive Definite Functions and Related Topics, Springer-Verlag, New York-BerlinHeidelberg-Tokyo, 1984.

5. Berg, C. and Maserick, P. H., Exponentially bounded positive definite functions, Illinois J. Math. 28 (1984), 162-179.

6. Berkson, E., Semigroups of scalar-type operators and a theorem of Stone, Illinois J. Math. 10 (1966), 345-352.

7. Diestel, J. and Uhl, J. J. Jr., Vector Measures, Math. Surveys 15 (1977).

8. Dinculeanu, N., Vector Measures, Pergamon Press, New York, 1967.

9. Dodds, P. G. and Ricker, W. J., Spectral measures and the Bade reflexivity theorem, J. Funct. Anal. 61 (1985), 136-163.

10. Dodds, P. G., de Pagter, B. and Ricker, W. J., Reflexivity and order properties of scalar-type spectral operators in locally convex spaces, Trans. Amer. Math. Soc. 293 (1986), 355-380

11. Dunford, N. and Schwartz, J. T., Linear Operators III: Spectral Operators, Wiley Interscience, New York, 1971.

12. Falb, P. L. and Haussmann, U., Bochner's theorem in infinite dimensions, Pacific J. Math. 43 (1972), 601-618.

13. Gillespie, T. A., Boolean algebras of projections and reflexive algebras of operators, Proc. London Math. Soc. 37 (1978), 56-74.

14. Ionescu Tulcea, C., Spectral representation of certain semigroups of operators, J. Appl. Math. Mech. 8 (1959), 95-109.

15. Kantorovitz, Sh., On the characterization of spectral operators, Trans. Amer. Math. Soc. 111 (1964), 152-181.

16. Kantorovitz, Sh., Semigroups of Operators and Spectral Theory, Pitman Res. Notes Math. Ser. 330 (1995).

17. Kluvánek, I., Characterization of Fourier-Stieltjes transforms of vector and operator-valued measures, Czechoslovak Math. J. 17(92) (1967), 261-276.

18. Kluvánek, I., Fourier transforms of vector-valued functions and measures, Studia Math. 37 (1971), 1-12.

19. Kluvánek, I. and Knowles, G., Vector Measures and Control Systems, North Holland, Amsterdam, 1976.

20. Lange, R. and Nagy, B., Semigroups and scalar-type operators in Banach spaces, J. Funct. Anal. 119 (1994), 468-480.

21. Lanier, L. H. Jr., Semigroups of Spectral Operators, PhD Thesis, University of Illinois, Urbana, 1963.

22. Lumer, G., Spectral operators, Hermitian operators, and bounded groups, Acta. Sci. Math. (Szeged) 25 (1964), 75-85.

23. Maserick, P. H., Spectral theory of operator-valued transformations, J. Math. Anal. Appl. 41 (1973), 497-507.

24. Nagy, B., On Stone's theorem in Banach spaces, Acta. Sci. Math. (Szeged) 57 (1993), 207213.

25. Nguyen Van Thu, On additively correlated random variables, Bull. Acad. Polon. 23 (1975), 781-785.

26. Panchapagesan, T. V., Unitary operators in Banach spaces, Pacific J. Math. 22 (1967), 465475 . 
27. Panchapagesan, T. V., Semigroups of scalar-type operators in Banach spaces, Pacific J. Math. 30 (1969), 489-517.

28. Räbiger, F. and Ricker, W. J., $C_{0}$-semigroups and cosine families of linear operators in hereditarily indecomposable Banach spaces, Acta. Sci. Math. (Szeged) 64 (1998), 697706.

29. Ressel, P., On additively correlated random processes, In: Prediction Theory and Harmonic Analysis, The Pesi Masani Volume, V. Mandrekar \& H. Salehi (eds.), North Holland, Amsterdam, 1983, pp. 253-261.

30. Ressel, P. and Ricker, W. J., Semigroup representations, positive definite functions and abelian $C^{*}$-algebras, Proc. Amer. Math. Soc. 126 (1998), 2949-2955.

31. Ricker, W. J., Semigroups of operators and an application to spectral theory, Math. Proc. Cambridge Philos. Soc. 96 (1984), 143-149.

32. Ricker, W. J., Well-bounded operators of type (B) in a class of Banach spaces, J. Austral. Math. Soc. Ser. A 42 (1987), 399-408.

33. Ricker, W. J., Integral transforms of vector measures on semigroups with applications to spectral operators, Semigroup Forum 45 (1992), 342-363.

34. Schaefer, H. H., Topological Vector Spaces (3rd Ed.), Springer-Verlag, New York-BerlinHeidelberg, 1971.

35. Schaefer, H. H., Banach Lattices and Positive Operators, Springer-Verlag, New York-BerlinHeidelberg, 1974.

36. Sourour, A. R., Semigroups of scalar-type operators on Banach spaces, Trans. Amer. Math. Soc. 200 (1974), 207-232.

37. Sourour, A. R., On groups and semigroups of spectral operators on a Banach space, Acta. Sci. Math. (Szeged) 36 (1974), 291-294.

38. Sz.-Nagy, B., Extension of linear transformations in Hilbert space which extend beyond this space, Appendix to: F. Riesz \& B. Sz.-Nagy, Functional Analysis, Ungar, New York, 1960.

39. Tweddle, I., Weak compactness in locally convex spaces, Glasgow Math. J. 9 (1968), 123-127.

MATH.-GEOGR. FAKULTÄT

KATHOLISCHE UNIVERSITÄT EICHSTÄTT

D-85071 EICHSTÄTT

GERMANY
SCHOOL OF MATHEMATICS

UNIVERSITY OF NEW SOUTH WALES

SYDNEY, N.S.W., 2052

AUSTRALIA

PERMANENT ADDRESS:

MATH.-GEOGR. FAKULTÄT

KATHOLISCHE UNIVERSITÄT EICHSTÄTT

D-85071 EICHSTÄTT

GERMANY 